
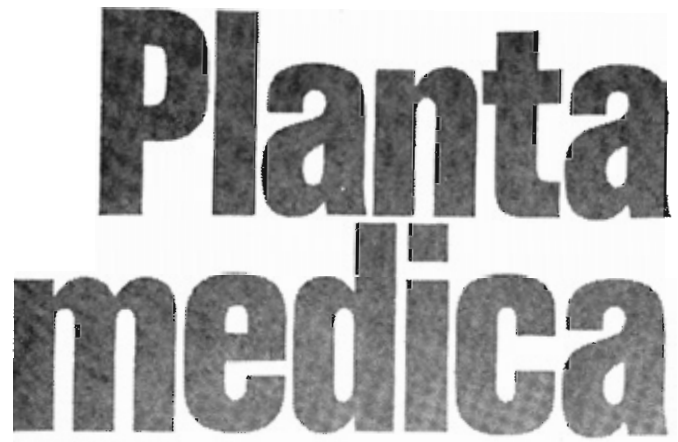

\title{
Journal of Medicinal
}

Plant Research

Editor-in-Chief E. Reinhard, Tübingen Pharmazeutisches Institut Auf der Morgenstelle 8 D-7400 Tübingen
Editorial Board

H. P. T. Ammon, Tübingen

W. Barz, Münster

E. Reinhard, Tübingen

O. Sticher, Zürich

$H$. Wagner, München

M. H. Zenk, München
Hippokrates Verlag

Stuttgart

Vol. 39

August 1980

No.

\section{Neue pharmakologisch interessante Naturstoffe}

\section{New Pharmacologically Interesting Natural Substances}

\section{Baumgarth}

Pharma-Forschung/Naturstoffe, E. Merck, Darmstadt, Bundesrepublik Deutschland

Herrn Prof. Dr. R. Tschesche zum 75. Geburtstag gewidmet

Key Word Index: Natural Substances; Structure; Pharmaçological Activities.

\section{Abstract}

Some of the most interesting pharmacologically active natural substances from plants (and some animals) are reviewed. Their structures and/or their activities were published during the years
1973-1979. The substances are discussed according to the main classes of natural compounds in the following order: Alkaloids and other Nitrogen-containg compounds; Terpenoids; Oxygen-Heterocycles; Glycosides; Compounds with other structures. 


\section{Einleitung}

1973 erschien eine Übersicht unter dem Titel "Neue pharmakologisch interessante Inhaltsstoffe höherer Pflanzen" [1]. Im Titel des vorliegenden Úberblicks, der sich chronologisch daran anschließt, ist der Begriff „Inhaltsstoffe höherer Pflanzen“ durch „Naturstoffe“ ersetzt. Durch die folgenden Definitionen der Titelbegriffe „Neu“, "pharmakologisch interessant" und "Naturstoffe" sind gleichzeitig die Auswahlkriterien gegeben:

"Neu" soll sich sowohl auf die Verbindungen als auch auf die Wirkungen beziehen, d. h., daß bei einem Naturstoff, der schon Jahre, in einigen Fällen Jahrzehnte auch in seiner Struktur bekannt ist, das erstmalige Auffinden einer pharmakologisch interessanten Wirkung, oder umgekehrt, daß erst die neuerdings erfolgte Korrelation eines schon länger bekannten Wirkprinzips mit einer neuen chemischen Struktur die Aufnahme in diese Ubersicht rechtfertigen.

Als "pharmakologisch interessant " werden Verbindungen angesehen, bei denen Wirkung, Wirkungsprofil oder Nebenwirkung besonders auffällig sind oder eine nicht ungewöhnliche Wirkung an einen neuen Strukturtyp geknüpft ist. Die pharmakologische Wirkung sollte irgendwie belegt sein, z. B. durch Ängabe der Prüfmodelle und Wirkungsstärke oder wenigstens durch Vergleich mit Standardsubstanzen, toxikologische Angaben etc. Naturstoffe, die in ihrer Struktur und/oder Wirkung bereits bekannten Verbindungen sehr ähnlich sind (z. B. neue Herzglykoside, Flavanoide) werden ebensowenig erfaßt wie solche, die bisher nur toxikologisches Interesse beanspruchen. Nicht behandelt werden Antibiotika und Zytostatika; zum einen würde die Zahl ihrer neuen Vertreter den Rahmen dieses Úberblicks sprengen, zum anderen existieren genügend neuere Reviews über beide Gebiete (z. B. [2] [5]).

Unter "Naturstoffen“ sind niedermolekulare, chemisch definierte Verbindungen aus höheren und niederen Pflanzen sowie aus tierischen Organismen zu verstehen. Gemische (Extrakte) müssen wiederum aus Kapazitätsgründen genauso ausgenommen werden wie Verbindungen, deren Strukturen noch unbekannt sind.

In meinem letzten Úberblick [1] habe ich 44 Verbindungen vorgestellt, von denen $\mathrm{m}$. W. keine bisher als. Wirkstoff eingeführt wurde, und nur ganz wenige bis heute von pharmakologischem oder klinischem Interesse geblieben sind. Das könnte den Eindruck erwecken, als seien Naturstoffe als potentielle pharmazeutische Wirkstoffe bedeutungslos. Dagegen seien einige Zahlen gestellt, die zumindest die bisherige Bedeutung von Naturstoffen als Pharma-Wirkstoffe beleuchten können:

FARNSWORTH hat z. B. für 1973 bei den in den USA über Apotheken und gegen Rezept insgesamt abgegebenen Präparaten den Anteil der Naturstoffe (inkl. Extrakte) enthaltenden Präparate ermittelt: Er betrug insgesamt $41,2 \%$, davon entfielen auf Naturstoffe aus höheren Pflanzen 25,2\%, aus Mikroorganismen $13,3 \%$ und aus Tieren 2,7 \% [6].

In den Jahren 1961-1977 wurden weltweit 1330 Pharma-Wirkstoffe neu eingeführt; die Zahl entstammt Publikationen des Bundesverbandes der Pharmazeutischen Indurstrie $[7,8]$. Ermittelt man da- 
von den Anteil der Naturstoffe, wobei partialsynthetische Antibiotika ebenso eingeschlossen sind wie einfache Derivate (Ester, Ather, Salze etc.) sonstiger $\mathrm{Na}$ turstoffe, unabhängig davon, ob die Grundmoleküle heute aus der Natur gewonnen oder synthetisch hergestellt werden, so ergibt sich folgendes Bild:
Erforschung der Fauna und Flora bestimmter geographischer und ökologischer Bereiche.

Hierfür seien zwei Ansatzpunkte erwähnt:

Das Bundesministerium für Forschung und Technologie fördert seit kurzem im Rahmen des Biotechnologie-Programms

\section{Tabelle I}

\begin{tabular}{llll}
$\begin{array}{l}\text { Wirkstoffe } \\
\text { insgesamt }\end{array}$ & Antibiotika & $\begin{array}{l}\text { Sonstige } \\
\text { Naturstoffe }\end{array}$ & $\begin{array}{l}\text { Naturstoffe } \\
\text { insgesamt }\end{array}$ \\
\hline $1961-73$ & $76=7,1 \%$ & $55=5,2 \%$ & $131=6,8 \%$ \\
\hline $1974-77$ & $21=7,9 \%$ & $39=14,8 \%$
\end{tabular}

Bei diesen Relationen ist natürlich nicht berücksichtigt, daß ein großer Teil der Wirkstoffe auf Naturstoff-Vorbilder zurückgeht. Außerdem sagen die Zahlen aus beiden Untersuchungen selbstverständlich nichts über den medizinischen und kommerziellen Wert der NaturstoffPräparate aus.

Im Úberblick von 1973 wurden drei Prinzipien für die Auffindung potentiell in der Natur vorhandener Wirkstoffe herausgestellt:

Untersuchung von in der Volksmedizin verwendeter Pflanzen bzw. deren Extrakten.

Dieses Verfahren betrifft im wesentlichen höhere Pflanzen, ist aber, wie die späteren Beispiele zeigen werden, recht erfolgversprechend. Die Bedeutung dieses Ansatzpunktes wird durch zahlreiche Symposien belegt.

1979 fand z. B. in Rom ein internationales Seminar unter dem Thema "Plants in Traditional Medicine" statt [9].
Forschungs- und Entwicklungsarbeiten zum Thema "Pharmakologisch wirksame Substanzen aus tropischen Heilpflanzen".

Hierzu gehört auch die zunehmend erfolgversprechende sog. Marine Pharmakologie. Das Interesse der Industrie daran hat sich in letzter Zeit z. B. in der Errichtung eines eigenen Instituts für Meerespharmakologie in Sydney durch Hoffmann-La Roche manifestiert.

Breite systematische Untersuchung ganzer Pflanzengattungen oder -familien, meist in einer bestimmten Indikationsrichtung. Hierzu gehört das durch die USRegierung geförderte Programm zur Auffindung von Zytostatika in der Natur.

Uber diese Ansatzpunkte hinaus kommt der chemischen Abwandlung wirksamer Naturstoffe allmählich die gleiche Bedeutung zu wie in der synthetisch-pharmazeutischen Chemie schon immer. Erinnert sei an einige erfolgversprechende Beispiele aus neuerer Zeit 
[10]: die Cannabinoid-Analoga [11,12], die partialsynthetischen Mutterkornalkaloide [13,14], die Azidomorphine [10]. $\mathrm{Da}$ es hierüber mittlerweile z. T. umfangreiche Ubersichten gibt [10-14], soll später an einem aktuelleren Beispiel gezeigt werden, wie man durch Veränderungen des natürlichen Grundmoleküls zu stärkeren, selektiveren oder sogar in ganz anderen Richtungen wirkenden Substanzen gelangen kann.

Im UUberblick des Autors von 1973 wurden die Verbindungen nach ihren pharmakologischen Hauptwirkungen gegliedert, diesmal werden die Naturstoffe aufgrund ihrer Strukturen innerhalb $\mathrm{Na}$ turstoff-Klassen besprochen.

Zwischen der ersten Úbersicht des Autors [1] und der vorliegenden, die die Literatur bis Herbst 1979 berücksichtigt, erschienen Zusammenstellungen mit ähnlicher Thematik: eine über die in der Literatur von 1974/75 berichteten biologisch aktiven Substanzen pflanzlichen Ursprungs [15] und der Sammelband mit den Plenarvorträgen vom First International Congress on Medicinal Plant Research (München 1967) [16]; darin die für dieses Thema besonders interessanten Kapitel über Alkaloide [17], Terpenoide [18], Saponine [19] und indische Arzneipflanzen [20]. Úberschneidungen mit diesen Arbeiten wurden vermieden. $\mathrm{Pa}$ tente und Kongreß-Abstracts wurden nur zitiert, wenn keine andere äquivalente Literaturstelle vorlag.

\section{Alkaloide und andere $\mathbf{N}$-haltige Verbindungen}

Unter den Alkaloiden, die lange Zeit als wichtigste Klasse von Naturstoffen mit auffallenden physiologischen Wirkungen galten, finden sich nach wie vor die meisten Verbindungen mit überraschenden Strukturen und/oder Wirkungen.

\section{Aminosäuren und Amide}

Von den biogenetischen Vorläufern der Alkaloide, den Aminosäuren, haben in letzter Zeit einige neue Vertreter aufgrund ihrer physiologischen Eigenschaften ebenso Beachtung gefunden, wie einige Amide.

Im Jahre 1975 berichteten japanische Autoren über Isolierung [21], Strukturaufklärung [22] und Synthese [23] einer neuen Aminosäure, Quisqualinsäure [I], die einige Jahre zuvor in der Frucht von Quisqualis indica (Combretaceae) zusammen mit anderen Aminosäuren gefunden worden war. Eine gewisse strukturelle Ähnlichkeit dieser heterocyclischen Aminosäure mit Glutaminsäure veranlaßte Pharmakologen bei der japanischen Firma Nippon Kayaku, die Verbindung auf eine mögliche Wirkung am neuromuskulären Knoten des Flußkrebses sowie an spinalen Neuronen von Frosch und Ratte zu untersuchen, denn L-Glutaminsäure gilt allgemein als wesentlicher Transmitter bei der Erregung an dieser Membran [24]. Applikation von Quisquatiasälre bewirkt eine starke Depolarisierung der Muskelfaser, die um 2-3 Größenordnungen stärker als die von Glutaminsäure ist, und induziert eine Desensibilisierung des Rezeptors für L-Glutaminsäure. Einige weitere, der Quisqualinsäure strukturell ähnliche natürliche Aminosäuren (wie z. B. Kaininsäure, Domoinsäure) haben neurophysiologisch analoge Eigenschaften [25]. Solche Verbindungen, die spezifische 
Antagonisten synaptischer und chemischer Erregungen sind, erlangen zunehmende Bedeutung in der experimentellen Pharmakologie.

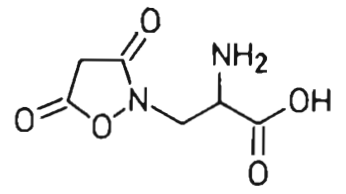

I Quisqualinsäure<smiles>CC1CS(=O)CC(C(=O)O)N1</smiles>

II Cycloalliin

Seit einiger Zeit ist die fibrinolytische Aktivität der Speisezwiebel (Allium cepa, Liliaceae) und ihrer Extrakte bekannt [26]. Sowohl das Wasserdampf-Destillat als auch ein Äther-Extrakt verstärken die Fibrinolyse im Blut ohne FibrinogenDepression [27]. Die Analyse der Extrakte führte zu einer Reihe in größerer Konzentration vorhandener S-haltiger Verbindungen mit fibrinolytischen $\mathrm{Ei}$ genschaften, wie 3,4-Dimethylthiophen, Propyl-allyl-disulfid, S-Methyl- und SPropyl-cysteinsulfoxid, deren sehr intensiver und langanhaltender Geruch aber eine klinische Anwendung ausschließt. Dagegen ist die im alkoholischen Extrakt vorkommende S-haltige Aminosäure Cycloalliin (II), die schon VIRTANEN 1959 beschrieben hatte, nahezu geruchlos [28]. Die Verbindung besitzt indirekte fibrinolytische Aktivität: In vitro war die
Verbindung unwirksam (keine Erhöhung der Euglobulin-Lysis-Zeit); an Probanden bewirkte eine einmalige orale Gabe von $250 \mathrm{mg}$ signifikante Verstärkung der Fibrinolyse (gemessen an der Euglobulin-Lysis-Zeit), hatte aber keinen Effekt auf die Plättchenaggregation [29]. Eine Erklärungsmöglichkeit ist, daß die Substanz die Blutpfropfen unter Offnung von S-S-Querverbindungen zwischen Fibrinmolekülen durch eine Disulfid-Austausch-Reaktion schwächt und daher einer natürlichen Fibrinolyse zugänglicher macht.

Seit längerem ist bekannt, daß beim Verzehr des grauen Tintlings, Coprinus atramentarius (Basidiomycetae), einem in Nordamerika beheimateten und als eßbar geltenden Pilz; bei gleichzeitigem Alkoholgenuß Disulfiram-ähnliche toxische Manifestationen, wie Ubelkeit und Schläfrigkeit, auftreten [30]. Einer schwedischen [31] und einer amerikanischen Arbeitsgruppe [32] gelang unabhängig voneinander die Isolierung des toxischen Prinzips, Coprin genannt, und seine Strukturaufklärung als $\mathrm{N}^{5}-(1-\mathrm{Hy}-$ droxycyclopropyl)-glutamin (III). Letztere erfolgte u. a. durch Spaltung in Glutaminsäure und 1-Hydroxy-cyclopropylamin (IV), das nur in Form seines $\mathrm{Hy}-$ drochlorids oder seiner O-Alkylderivate stabil ist. Die schwedische Gruppe beschrieb auch Synthesen von III und IV $[33,34]$. Coprin ist der erste, ein Cyclopropanon-Aquivalent enthaltende $\mathrm{Na}$ turstoff und das erste beschriebene $\mathrm{N}$ Acyl-1-aminopropanol. Die Verbindung ist ein starker Acetaldehyd-Dehydrogenase-Inhibitor, jedoch nur in vivo; in vitro ist sie vollkommen wirkungslos. Somit sollte ein Metabolit das wirksame Prinzip sein [35]. In der Tat erwies sich 
das 1-Hydroxycylopropylamin (IV) als der eigentliche Wirkstoff. Tierexperimente zeigten, daß auch andere Hydroxy- und Alkoxy-cyclopropylcarboxamide den gleichen in vivo-Effekt zeigten wie Coprin und sein Hydrolyseprodukt. Coprin und seine Analoga sind von Interesse zur Behandlung des Alkoholismus. Coprin hat gegenüber Disulfiram den Vorteil, daß es die Dopamin-ß-hydroxylase nicht hemmt und damit einige Nebenwirkungen dieser Verbindung möglicherweise nicht aufweist.

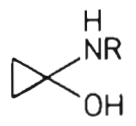

III Coprin $(R=\underbrace{N_{1}}_{\prod_{0}} \overbrace{0}^{H_{2}} O H)$

IV 1-Hydroxy-cyclopropylamin $(\mathrm{R}=\mathrm{H})$

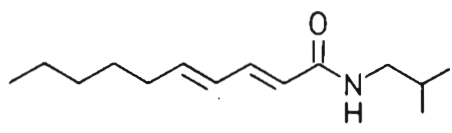

V „waxy alkaloid”

Die pharmazeutische Bedeutung des Pfeffers ist gering, obwohl in der volksmedizinischen Literatur verschiedene $P i$ per-Arten als Oxytocicum, Abortivum und Emmenagogum erwähnt sind. Neuere Untersuchungen in Indien über die postkoitale Antifertility-Aktivität von Extrakten verschiedener Piper-Arten an Ratten zeigten, daß die Aktivität am ausgeprägtesten im Petroläther-Extrakt von Piper officinarum und $P$. longum ist (Piper nigrum, das zusammen mit $P$. al- bum den Gewürzpfeffer darstellt, ist wirkungslos). Unter den darin vorkommenden Amiden, wie Piperin, Piplaritin, Filfilin und dem sogenannten "waxy alkaloid“ (V) (= N-Isobutyl-deca-trans-2trans-4-dienamid) [36], ist das letztere am effektivsten als Implantationshemmer und als frühes Abortivum (46\% Unterdrückung der Implantation und $82 \%$ der Schwangerschaft bei $100 \mathrm{mg} / \mathrm{kg}$ an der Ratte) [37]. Semisynthetische Analoga von Piperin und "waxy alkaloid“ zeigten deutlich schwächere Wirkungen.

Die Droge „Mao“ wird aus den oberirdischen Teilen bestimmter Ephedra-Arten (Ephedraceae) bereitet und in der orientalischen Medizin als schweißtreibendes Mittel sowie bei Husten, Fieber und Entzündungen angewendet. Seit dem Auffinden von (-)-Ephedrin (VI) im Jahre 1887, das später für die sympathomimetischen, vasokonstriktorischen und antiallergischen Eigenschaften der Droge verantwortlich gemacht werden konnte, wurden aus Ephedra-Blättern sein Norund sein Methyl-Analogon, die entsprechenden Analoga in der (+)-Pseudoephedrin-Reihe und andere Alkaloide, wie 3,4-Dimethyl- und 2,3,4-Trimethyl5-phenyloxazolidin isoliert. Eine japanische Arbeitsgruppe fand kürzlich mit dem Blatt-Extrakt von Ephedra intermedia antiinflammatorische Wirkung und isolierte als aktives Prinzip ein neues Alkaloid, das Ephedroxan [38]. Es handelt sich um das Oxazolidon VII, wie sich u. a. durch Synthese aus (-)-Ephedrin (VI) ergab. Die Verbindung war bereits 1959 synthetisiert worden; dies ist aber der erste Hinweis auf ihr natürliches Vorkommen. Ephedroxan wurde noch in einigen weiteren, aber nicht in allen Ephedrin-haltigen Ephedra-Arten gefun- 


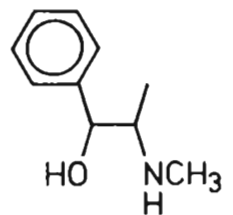

VI Ephedrin

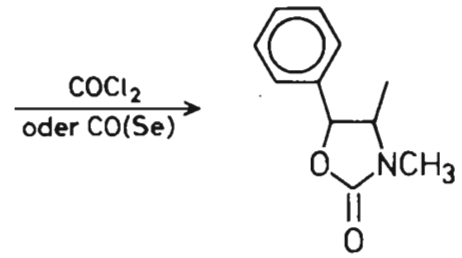

VII Ephedroxan

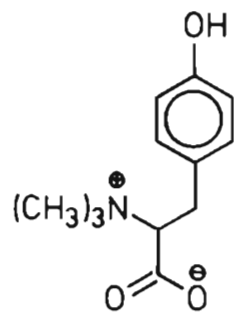

VIII Maokonin

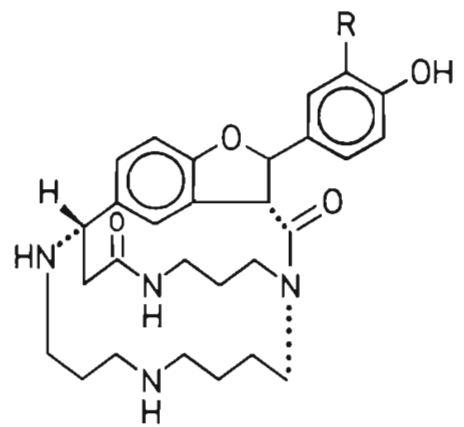

$\begin{array}{ll}\text { IX Ephedadrin } & A(R=\mathrm{H}) \\ B\left(\mathrm{R}=\mathrm{OCH}_{3}\right)\end{array}$ den. Da einerseits sein Gehalt darin gering ist $(0,0003-0,002 \%)$, andererseits seine antiinflammatorische Aktivität zwar in verschiedenen Modellen nachweisbar aber schwach sein soll (Einzelheiten liegen noch nicht vor), kann die ausgeprägte antiinflammatorische Aktivität der Droge nicht allein diesem Alkaloid zugeschrieben werden.

Dagegen sagt man der Droge "Maōkon ", den unterirdischen Teilen von Ephedra-Arten, gegensätzliche Wirkungen nach. Sie wurde in der orientalischen Medizin bei Schweißausbrüchen eingesetzt. Außerdem gab es einen Hinweis aus den zwanziger Jahren auf die hypotensive Wirkung des Wurzel-Extrakts. Dies war für den gleichen Arbeitskreis, der sich auch mit "Maō“ beschäftigte, $\operatorname{der}$ Anlaß zur näheren Untersuchung der
Droge [39]. Im vergangenen Jahr isolierte man aus dem Methanol-Extrakt zusätzlich eine basische Substanz, bei der es sich um Tyrosin-betain handelte, wie sich durch Methylierung von L-Tyrosin beweisen ließ. Die Verbindung wurde zum ersten Mal im Pflanzenreich gefunden und Maokonin (VIII) genannt. Uberraschenderweise zeigte Maokonin an der narkotisierten Ratte eine hypertensive Wirkung. Es wirkt damit ähnlich wie Ephedrin, das Hauptalkaloid der oberirdischen Teile der Pflanze, und entgegengesetzt wie die aus den unterirdischen Teilen gewonnene Rohdroge, die damit neben Maokonin noch ein oder mehrere hypotensive Prinzipien enthalten muß.

Solche wurden im gleichen Arbeitskreis durch weitere Fraktionierung des 
Extrakts auch gefunden und Ephedadrin $A$ und $B$ (IX, X) genannt $[40,41]$. Chemisch handelt es sich bei diesen Alkaloiden, wie sich durch Aufklärung der $\mathrm{Hy}$ drolyseprodukte, Röntgenstrukturanalyse des Ephedadrins A und Vergleich der Spektren beider Verbindungen zeigen ließ, um relativ komplizierte Peptide aus dem Tetramin Spermin und jeweils einer phenolischen Dicarbonsäure, deren Biogenese im Falle des Ephedadrins A aus 2 Molekülen p-Cumarsäure, im Falle des Ephedadrins B aus je einem Molekül pCumarsäure und Ferulasäure angenommen wird. Am gleichen Tiermodell wie Maokonin geprüft, führte die i.v.-Gabe von $1,5-1,8 \mathrm{mg} / \mathrm{kg}$ Ephedadrin A bzw. $2,0 \mathrm{mg} / \mathrm{kg}$ Ephedadrin $B$ zu einem deutlichen Blutdruckabfall. Das Vorkommen der entgegengesetzt wirkenden Prinzipien Maokonin und Ephedadrin A bzw. $B$ in der gleichen Pflanze zeigt, wie vorsichtig man mit der Korrelation von volksmedizinischen Befunden, Wirkungen von Extrakten und einzelnen Wirkprinzipien sein sollte.

\section{Benzylisochinolin-Alkaloide}

Die in diesem Kapitel zusammengefaßten Alkaloide (Proaporphine, ein Aporphin-Folgeprodukt, Bisbenzylisochinoline) sind mit einer Ausnahme schon länger bekannt, aber erst in letzter Zeit pharmakologisch besonders aufgefallen.

Während eines pharmakologischen Screenings brasilianischer Pflanzen fiel die ZNS-Aktivität von Extrakten aus Ocotea glaziovii (Lauraceae) auf, die mit der razemischen Form des schon 1964 isolierten Proaporphin-Alkaloids Glaziovin (XI) [42] korreliert werden konnte [43,44]. 1975 wurden zwei Totalsynthe- sen von (+)-Glaziovin publiziert $[45,46]$. In verschiedenen Tierversuchen erwies sich das Alkaloid in oralen Gaben zwischen 0,1 und $1 \mathrm{mg} / \mathrm{kg}$ als relativ untoxischer Tranquillizer $\left(\mathrm{LD}_{50}\right.$, Maus und Ratte p.o. um $540 \mathrm{mg} / \mathrm{kg}$ ) [47]. In einer klinischen Doppelblindstudie wirkte es ähnlich anxiolytisch wie Diazepam, bewirkte aber im Gegensatz dazu keine Asthenie oder Schläfrigkeit [48]. Seine besonders starke anxiolytische Aktivität im Gastrointestinal-Trakt veranlaßte die Prüfung auf antiulcerogene Wirkung [49]. Diese war bei verschiedenen experimentell hervorgerufenen Geschwüren an Ratten und Meerschweinchen bei parenteraler Gabe von $5-10 \mathrm{mg} / \mathrm{kg}$ stärker als die von Sulpirid mit $10 \mathrm{mg} / \mathrm{kg}$.

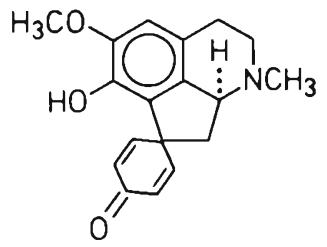

XI Glaziovin

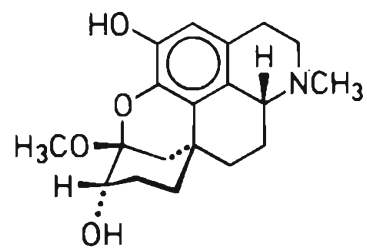

XII Kesselringin

Colchicum kesselringii (Liliaceae) enthält wie andere Colchicum-Arten Alkaloide mit Tropolon-Ring. Russische Autoren isolierten aber daraus auch ein Nicht-Tropolon-Alkaloid und nannten es Kesselringin (XII) [50,51], dessen 
Strukturaufklärung mit Festlegung der absoluten Konfiguration 1977 gelang $[52,53]$. Es handelt sich um einen Vertreter der relativ seltenen Homoproaporphin-Alkaloide, bei dem ein zusätzlicher Ringschluß durch Acetalbildung zwischen dem aromatischen und dem SpiroRing erfolgt ist. Kesselringin zeigte in Dosen von $10-100 \mathrm{mg} / \mathrm{kg}$ an Mäusen und Ratten verschiedene ZNS-Wirkungen, wie Verlängerung der Morphin-Analgesie, Potenzierung der Wirkung von Amytal-Natrium und Chlorpromazin, Inhibierung der Orientierungsreaktion sowie der spontanen motorischen und der corticalen EEG-Aktivität; daneben wurde ein gewisser hypothermer Effekt beobachtet. Die $\mathrm{LD}_{50}$ betrug an der Ratte $380 \mathrm{mg} / \mathrm{kg}$ [54].

Bei dem im oberen Amazonas-Tal von Peru wachsenden Baum "Sangre de grado" handelt es sich um die Euphorbiacee Croton lechleri. Durch Anschneiden der Rinde läßt sich ein roter viskoser Saft gewinnen, der bei den peruanischen Indianern gegen verschiedene Krankheiten, einschließlich Rheumatismus, Anwendung findet. Aus diesem Saft wurde kürzlich Taspin (XIV) isoliert [55], das schon in den fünfziger Jahren aus Leontice eversmanii (Berberidaceae) [56], später auch aus $L$. albertii [57], gewonnen und in seiner Struktur geklärt worden war $[58,59]$. Es handelt sich um ein Alkaloid ungewöhnlicher Struktur, das offensichtlich in naher biogenetischer Beziehung zu den Aporphinen steht: aus einem solchen, dem weit verbreiteten Magnoflorin (XIII), könnte es durch enzymatischen Hofmann-Abbau, Oxidation der neu entstandenen Doppelbindung und anschließenden Lactonisierung entstehen; diese Reaktionsfolge gelang inzwischen

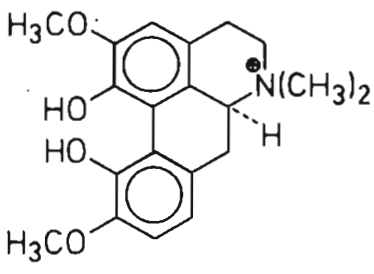

XIII Magnoflorin

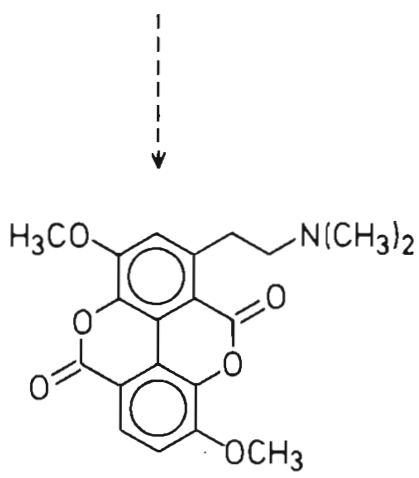

XIV Taspin

auch chemisch [60]. Taspin ist in Form seines Hydrochlorids an der Ratte in verschiedenen Testmodellen antiphlogistisch wirksam [55]: Im Carrageenininduzierten Pfotenödem ist es mit einem oralen $\mathrm{ED}_{50}$-Wert von $58 \mathrm{mg} / \mathrm{kg} \mathrm{3-4mal}$ aktiver als Phenylbutazon. Im Granulombeutel-Text inhibiert es mit einer Tagesdosis von $20 \mathrm{mg} / \mathrm{kg}$ innerhalb einer Woche die Granulombildung gleich stark wie Indomethacin mit $1 \mathrm{mg} / \mathrm{kg}$. In der gleichen Dosierung reduziert es im Adjuvans-Arthritis-Modell das Pfotenvolumen ähnlich wie Indomethacin mit $1 \mathrm{mg} /$ $\mathrm{kg}$. Dabei liegen diese Dosierungen deutlich unter der toxischen $\left(\mathrm{LD}_{50}\right.$ Ratte, oral $518 \mathrm{mg} / \mathrm{kg}$ für die Einzeldosis und $100 \mathrm{mg} / \mathrm{kg}$ für 7 Tagesdosen).

Cepharanthin (XV), ein Bisbenzylisochinolin-Alkaloid vom Biscoclaurin- 

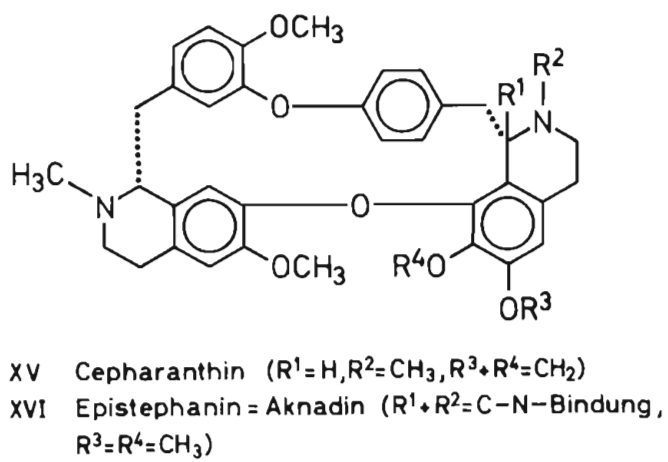

Typ, findet sich in Wurzeln und Stamm von Stephania cepharantha und S. sasakii (Menispermaceae) und wurde schon bald nach seiner Strukturaufklärung [61] mit Erfolg bei Alopezie und später in Japan gegen Tuberkulose angewendet. Nach neueren Experimenten in Japan blockiert Cepharanthin die Freisetzung biogener Amine, wie Histamin und Serotonin, aus Ratten-Mastzellen und stimuliert die Antikörper-Produktion [62]. In einer klinischen Studie an 50 Patienten mit Symptomen allergischer Rhinitis (Hydrorrhea, Ptarmus, Obstruktion) wurden 2 22 Wochen lang täglich $30 \mathrm{mg}$ des Alkaloids verabreicht; die Effektivität lag bei $68 \%$.

Stephania bernandifolia ist eine andere Alkaloid-reiche Menispermacee, die in Indien bei Diarrhoe, Dyspepsie und Krankheiten der Harnorgane eingesetzt wird. Moza und Mitarbeiter isolierten daraus u. a. ein nicht näher charakterisiertes Bisbenzylisochinolin-Alkaloid, das sie Aknadin nannten[63]. Bei einer neueren Untersuchung [64] erwies sich dieses als identisch mit dem schon 1954 in seiner Struktur geklärten Oxyacanthin-Vertreter Epistephanin (XVI) [65], das bereits früher in Stephania japonica gefunden worden war. Gleichzeitig fand man, daß die Gesamtalkaloid-Fraktion aus den oberirdischen Teilen der Pflanze an der sympathischen Nervenendigung wie Guanethidin wirkte. Durch Untersuchungen an verschiedenen isolierten Organen ließ sich diese Wirkung auf das Epistephanin zurückführen. Seine hypotensive Wirkung beträgt allerdings nur etwa $1 / 10$ von der des Guanethidins, setzt später ein und hält kürzer an. Parallele Dosis-Wirkungskurven beider Verbindungen lassen auf den gleichen Wirkungsmechanismus schließen [64].

\section{Indol-Alkaloide}

Schon in meiner letzten Ubersicht habe ich auf das Mitragynin (XVII) aus Mitragyna speciosa (Rubiaceae), ein Indol-Alkaloid vom Corynanthein-Typ, hingewiesen. Es handelt sich um eine analgetisch wirkende Verbindung, die mit keinem der bekannten Analgetika strukturell verwandt ist und einige der unangenehmen Nebenwirkungen der Analgetika vom Morphin-Typ nicht besitzen soll. Aus diesen Gründen hat die Firma Smith, Kline und French an der Chemie und Biologie dieser Substanz weiter gear- 


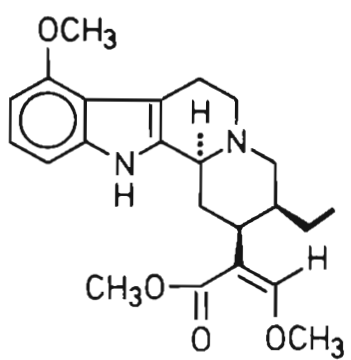

XVII Mitragynin

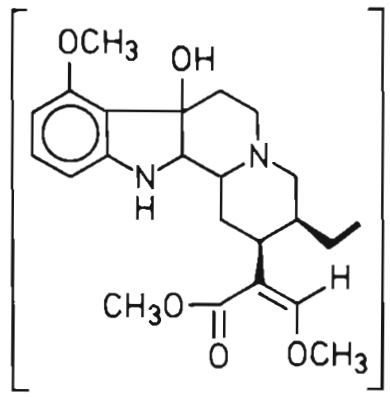

XVIII

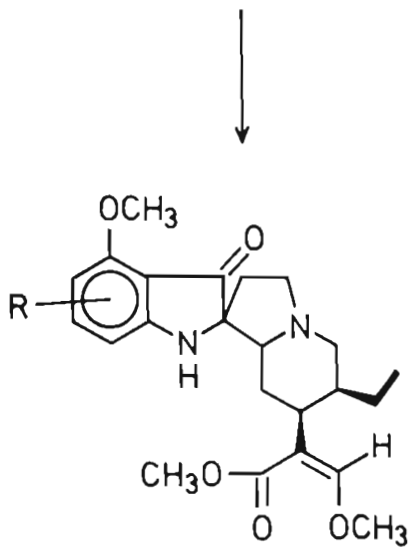

XIX,XX PseudoindoxyI Metaboliten $\langle\mathrm{R}=\mathrm{H}$ oder $\mathrm{OH}$ ) beitet und dabei Mitragynin u.a. einer Biotransformation durch einen Helminthosporum-Pilz unterworfen [66]. Die beiden Hauptmetaboliten, deren Mischung gleichartig wie die Ausgangssubstanz aktiv war, wurden abgetrennt: Die aktivere Verbindung war im Tierversuch (D'Amour-Smith-Test) 10fach stärker analgetisch wirksam als Mitragynin, sowohl bei oraler als auch bei i.p.-Gabe. Der zweite Metabolit zeigte in diesem Test nur noch 1/3 der Mitragynin-Aktivität. Spektroskopische Untersuchungen wiesen den aktiveren Metaboliten als Pseudoindoxyl-Derivat des Mitragynins (XIX) den weniger aktiven als dessen im Benzolring hydroxyliertes Analogon (XX) aus. Der nicht-hydroxylierte Metabolit ließ sich auch neben anderen Produkten durch Umsetzung von Mitragynin mit Bleitetraacetat und anschließend mit Na-methylat gewinnen. Die mikrobiologische Transformation scheint nach einem Oxidations-Hydroxylierungsmechanismus zu verlaufen. Die Spekulation, daß die Produkte ohne Mitwirkung des Pilzes durch einfache Luftoxidation während der Fermentation entstehen, wird unwahrscheinlich durch das Auftreten des Ring-hydroxylierten Metaboliten, der bei chemischer Oxidation nicht entsteht, wogegen sich das biosynthetisch postulierte Zwischenprodukt (XVIII) nachweisen läßt. Der wirksamere Mitragynin-Metabolit ist ein gutes Beispiel dafür, wie man durch einfache Umwandlung eines Naturstoffs zu einem, in diesem Fall gleichartig aber stärker wirkenden Produkt kommen kann. Für die Änderung der Wirkrichtung durch Abwandlung werden bei den Pyrrolizidin-Alkaloiden noch Beispiele gegeben. 
Coronaridin, das in verschiedenen $\mathrm{Ta}$ bernaemontana- und Ervatamia-Arten (Apocynaceae) vorkommt [67,68], gehört zur Klasse der Ibogain-Alkaloide und erwies sich als Methoxycarbonylibogamin (=Desmethoxy-voacangin) (XXI) [69]. Es besitzt u. a. eine gewisse analgetische Wirkung ohne sonstige zentrale Effekte [68]. In zwei Arbeitskreisen (Fa. Lederle und Central Drug Research Institute I.ucknow) wurde es in letzter Zeit von neuem interessant durch Auffindung seiner beachtlichen AntifertilityWirkung $[67,70]$. Die Verbindung zeigte im Tierversuch schwache östrogene Wirkung, besitzt aber keine sonstigen Hormon-, Antihormon- oder uterotonischen Aktivitäten. Lediglich eine partielle Hemmung der Oxytocin-induzierten Uterus-Reaktion wurde festgestellt. Verabreichte man graviden Ratten zwischen dem 1. und 5. Tag der Tragzeit $30 \mathrm{mg} / \mathrm{kg}$ Coronaridin (als Hydrochlorid) täglich per os, so kam es verläßlich zu einem Schwangerschaftsabbruch. Bei Behandlung zwischen dem 6 . und 8. Tag war der

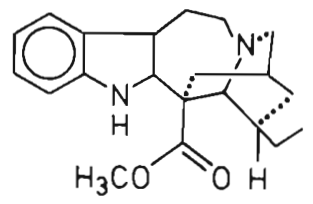

XXI Coronaridin

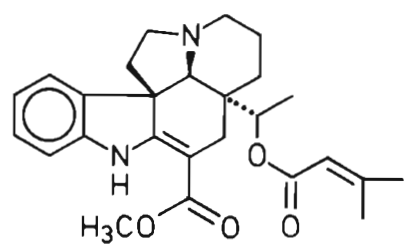

XXII Echitovenidin
Effekt nicht mehr $100 \%$ ig. Die Kontrazeption ist ausschließlich auf die östrogene Wirkung des Alkaloids zurückzuführen. Das mit Coronaridin eng verwandte Voacangin (= Methoxy-coronaridin) war in der gleichen Versuchsanordnung unwirksam.

Echitovenidin (XXII), das Hauptalkaloid in den Früchten von Alstonia venenata (Apocynaceae), ist ein Indol-Alkaloid vom Vindolin-Typ, in dem der ganze rechte Teil des Moleküls biogenetisch aus einer Monoterpen Einheit stammt [71]. Psychopharmakologische Studien, die Beobachtungstests an Mäusen und Ratten (i.p.) und Hunden (i.v.), Effekte auf eine Reihe von Pharmaka-induzierten Reaktionen sowie Bestimmung der $\mathrm{MAO}-\mathrm{Hemmer}$-Aktivität in vitro (unter Verwendung von mitochondrialen Enzymen aus Ratten-Hirn) umfaßten, wiesen die Verbindung als ein Antidepressivum vom MAO-Hemmer-Typ aus, wobei Wirkungsprofil und -stärke ähnlich der von Nialamid waren [72]. Die Verwendung der Droge in der indischen Volksmedizin bei Geisteskrankheiten wird so leicht verständlich.

Aus Arten der Gattung Gardneria (Loganiaceae) wurden bisher etwa 18 Indol-Alkaloide isoliert. Das Hauptalkaloid, Gardneramin (XXIII) findet sich in allen bisher untersuchten Arten, während sein 18-Desmethyl-Analogon XXIV und das sogenannte Alkaloid I (XXV) in G. multiflora, Gardnerin (XXVI), Gardnutin (XXVII) und Hydroxygardnutin (XXVIII) in G. nutans vorkommen. Chemisch wurden die Gardneria-Alkaloide von $\mathrm{S}_{\mathrm{AKAI}}$ eingehend untersucht [73]. Auffallend an der Struktur des Gardneramins und der an- 


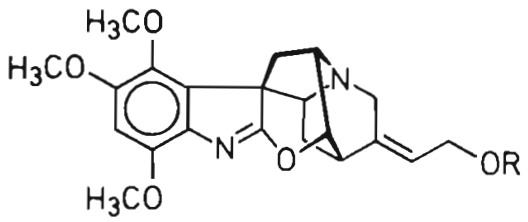

XXIII Gardneramin ( $\left.R=\mathrm{OCH}_{3}\right)$

XXIV 18-Desmethylgardneramin $(R=H)$

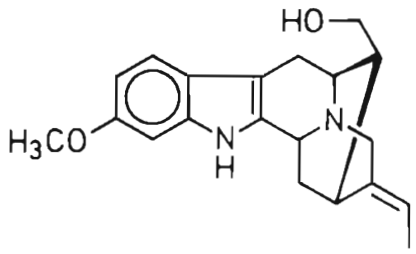

XXVI Gardnerin

deren beiden genannten Alkaloide vom Oxindol-Typ (XXIV, XXV) ist die unnatürliche Z-Konfiguration der Hydroxyäthyliden-Seitenkette [74], während man bisher bei den natürlich vorkommenden Indol-Alkaloiden E-Konfiguration der ungesättigten Seitenkette annahm, wie sie auch bei den hier angeführten Alkaloiden vom Sarpagin-Typ (XXVI - XXVIII) vorliegt. Da einerseits mit Gardneramin (XXIII) eine ganglienblockierende Wirkung gefunden worden war [75,76], andererseits Ganglienblokker in höheren Dosen Curare-ähnliche Wirkungen zeigen, wurden einige Gardneria-Alkaloide am Nerv-Muskel-Präparat (Rattenbein) untersucht: Dabei zeigte nur Gardneramin einen dem d-Tubocurarin und Hexamethonium ähnlichen Effekt, indem es die indirekt hervorgerufenen Kontraktionen unterdrückte (gleich stark wie Hexamethonium, aber länger

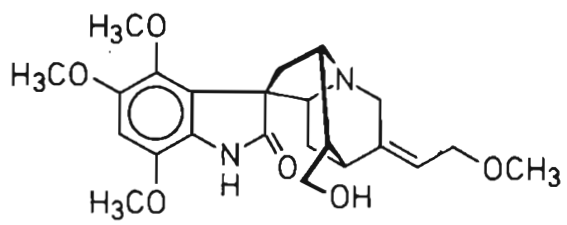

$X X V$ Alkaloid I

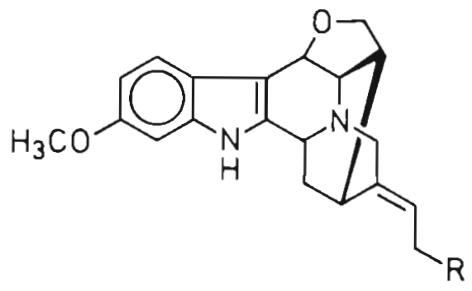

XXVII Gardnutin $(\mathrm{R}=\mathrm{H})$

XXVIII Hydroxygardnutin $(\mathrm{R}=\mathrm{OH})$

anhaltend), aber praktisch ohne Finfluß auf die direkt hervorgerufenen Kontraktionen war. Gardnerin (XXVI) verstärkte im Gegensatz dazu die indirekt und direkt ausgelösten Kontraktionen, während Gardnutin (XXVII) und Hydroxygardnutin (XXVIII) beide Effekte anhaltend unterdrückten [77]. Vergleichende Untersuchungen der 6 Gardneria-Alkaloide XXIII - XXVIII auf ihre ganglienblockierende Wirkung an Zervikalganglien von Kaninchen und Ratte ergab folgendes: Alle Verbindungen hoben das postganglionäre Aktionspotential vollständig, aber kurzfristig auf; die anschließende Erholung war ebenfalls vollständig. Die Wirkungsstärke nahm in folgender Reihenfolge ab: Gardnerin (XXVI) $\geqq$ Gardneramin (XXIII), AI $(\mathrm{XXV})>$ Gardnutin (XXVII) $>>\mathrm{Hy}-$ droxygardnutin (XXVIII), 18-Desmethylgardneramin (XXIV) und betrug 
beim Gardnerin und Gardneramin ca. $25 \%$ (Ratte) bis $50 \%$ (Kaninchen) der von Hexamethonium (bezogen auf einen Hemmeffekt von $60 \%$ ) [78].

Pyrrolizidin-und Chinolizidin-Alkaloide

Von den mehr als 100 bekannten Pyrrolizidin-Alkaloiden aus 40 Pflanzengattungen (insbesondere Senecio und Crotalaria) sind bisher im wesentlichen hepato- und pneumotoxische Eigenschaften bei Mensch und Vieh [79] und carcinogene, sowie von einigen spasmolytische [80], Curare-ähnliche und lokalanästhetische Wirkungen berichtet worden. Eine Ausnahme macht die klinische Anwendung zweier nicht-hepatotoxischer Pyrrolizidin-Alkaloide in der Sowjetunion, nämlich Diplacin und Platyphyllin wegen ihrer Curare- bzw. Atropin-ähnlichen Wirkung. Ein weiterer Vertreter dieser Klasse, der pharmakologisch in letzter Zeit Interesse gefunden hat, ist das Crotalaburnin (s. in [15]). Einige der toxischen Alkaloide können in hoher Ausbeute relativ leicht isoliert werden. In einem indischen Forschungsinstitut entschloß man sich daher, solche Vertreter als Ausgangsmaterialien für neue partialsynthetische Pyrrolizidine zu verwenden. Ausgewählt wurden das hepatotoxische Monocrotalin (XXX), das in Crotalaria retusa zu $8 \%$ vorkommt, und das untoxische, aus $C$. anagyroides stammende 1-Methylenpyrrolizidin (XXXI). Aus letzterem, seinem Hydrierungsprodukt Heliotridan (XXXIV), dem durch Verseifung von Monocrotalin erhältlichen Retronamin (XXXII) sowie dessen Hydrierungsprodukt Platynecin (XXXV) erhielt man durch chemische Modifizierung unter Vermeidung der offenbar für die toxische Wirkung verant- wortlichen Allyläther-Gruppierung insgesamt 120 Verbindungen innerhalb 5 verschiedener Substanzklassen. In jeder Klasse wurde eine der folgenden Hauptwirkungen gefunden: Lokalanästhetisch, hypotensiv, ganglienblockierend, muskelrelaxierend, spasmolytisch [81]. Ohne auf Chemie und Pharmakologie näher einzugehen, sei der jeweils potenteste Vertreter jeder Gruppe vorgestellt:

a) Quaternäre 1-Methylenpyrrolizidine, z. B. N-Isopropyl-1-methylen-pyrrolizidiniumbromid (XXIX):

Ganglienblocker ohne wesentliche Effekte am Herzen (Hund $5-10 \mathrm{mg} / \mathrm{kg}$ i.v. $50-70 \%$ Blutdruckabfall von 40 - 60', ausgeprägter beim hypertonen Tier) [82].

b) Retronamide, z. B. N-(p-Hydroxybenzoyl)-retronamin (XXXIII): hypotensiv (Hund i. v. 0,5 - $5 \mathrm{mg} / \mathrm{kg}$ : 37 - $75 \%$ Abfall, 12 - 150' anhaltend) [83].

c) Platynecin-diester, z. B. Platynecin7,9-disenecioat (XXXVI): lokalanästhetisch (1,3-bzw. 1,2mal Lignocain, topisch bzw. intradermal) [84].

d) N-Haloalkyl-heliotridaniumsalze, z. B. N-(2'-Bromäthyl)-beliotridaniumbromid (XXXVII): muskelrelaxierend (neuromuskuläre Blockade zwar schwächer, therapeutische Breite in Bezug auf Atmung größer als beim D-Tubocurarin) [85].

e) N-Acyl-heliotridaniumsalze, z. B. $N$ - (4-Phenylphenacyl) - heliotridaniumbromid (XXXVIII): spasmolytisch (4 - 5mal Papaverin) [86].

Erstaunlich sind bei diesen Abwandlungen zunächst relativ toxischer und pharmazeutisch wertloser Naturstoffe die Wirkungsdifferenzierung und das weit- 


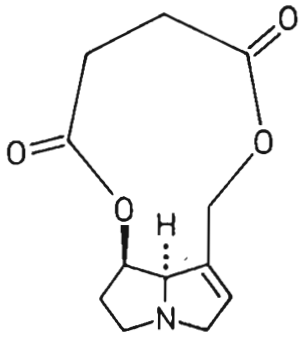

$x x x$ Monocrotalin

$$
\downarrow
$$

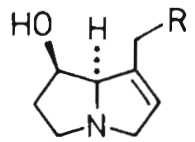

$x \times x I I$ Retronamin $(\mathrm{R}=\mathrm{OH})$

$X X X I I I N-(p-H y d r o x y b e n z o y I)-$ retron $=$ $\operatorname{amin}\left(\mathrm{R}=\mathrm{NHC}(\mathrm{O}) \mathrm{C}_{6} \mathrm{H}_{4} \mathrm{OH}\right)$

$$
\downarrow
$$<smiles>[R]O[C@H]1CCN2CC[C@H]([R2])[C@H]12</smiles>

$X X X V$ Platynecin $(R=H)$

$X X X V I$ Platynecin-disenecioat $\left(\mathrm{R}=\mathrm{C}(\mathrm{O}) \mathrm{CH}=\mathrm{C}\left(\mathrm{CH}_{3}\right)_{2}\right)$

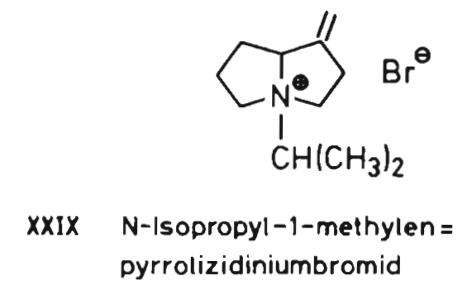

XXIX N-Isopropyl-1-methylen $=$ pyrrolizidiniumbromid
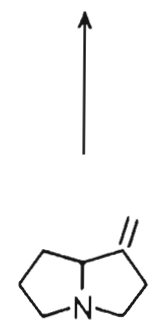

$X X X I \quad 1-M e t h y l e n-p y r r o l i z i d i n$

XXXIV Heliotridan
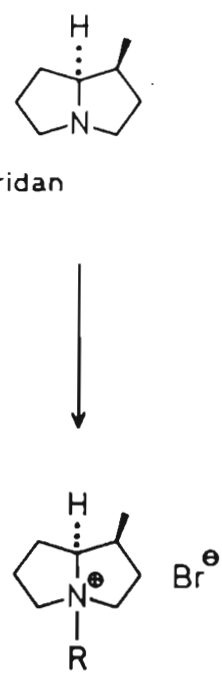

XXXVII $\mathrm{N}-\left(2^{\prime}-\right.$ Bromäthyl) - heliotridan = iumbromid $\left(\mathrm{R}=\mathrm{CH}_{2} \mathrm{Br}\right)$

$X X X V I I I N-(4-P h e n y \mid p h e n a c y l)-$ helio= tridaniumbromid

$\left(\mathrm{R}=\mathrm{C}(\mathrm{O}) \mathrm{C}_{6} \mathrm{H}_{6} \mathrm{C}_{6} \mathrm{H}_{5}\right.$ ) 
gehende Verschwinden der toxischen Eigenschaften.

Von Chinolizidin-Alkaloiden wieSpartein und 13-Hydroxylupanin sind hypotensive und antiarrhythmische Wirkungen bekannt. Veresterung von 13-Hydroxylupanin mit 2-Pyrrolcarbonsäure verstärkt beide Effekte deutlich. Der Ester, Calpurnin (XXXIX) genannt, wurde zusammen mit dem freien Alkohol in einigen Leguminosen (oberirdische Teile von Calpurnia subdecandra [87], verschiedene Teile von Cadia ellisiana und purpurea [88], Samen von Vir-

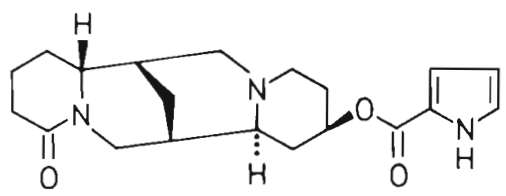

$x x x I x$ Calpurnin

gilia oroboides [89]) gefunden. Die Verbindung wurde kürzlich bei der Firma Hoechst unter der Chiffre HOE 933 pharmakologisch untersucht $[90,91]$ : Die blutdrucksenkende Wirkung erreichte bei Hunden, Affen und Ratten in Barbiturat-Narkose ihr Maximum mit 0;2 mg/ kg i.v., war aber bei nichtnarkotisierten Tieren deutlich schwächer. Die enterale Resorption war beim Hund gut: eine intraduodenale Dosis von $0,5 \mathrm{mg} / \mathrm{kg}$ erniedrigte bereits den Blutdruck. Außerdem besitzt Calpurnin starke antifibrillatorische (gezeigt mit $0,3 \mathrm{mg} / \mathrm{kg}$ i.v. bei unterkühlten Katzen) und antiarrhythmische Eigenschaften (mit $0,5 \mathrm{mg} / \mathrm{kg}$ bei Hunden mit k-Strophanthin-Arrhythmie nachweisbar). Am isolierten Meerschweinchen-Herz hemmte eine Dosis von nur $6 \mu \mathrm{g} /$ Herz das durch Aconitin und Digitoxin hervorgerufene Kammerflimmern. Auch die Toxizität des Digo- xins wurde durch vorherige i.v.-Gabe von $300 \mu \mathrm{g} / \mathrm{kg}$ Calpurnin vermindert. Allerdings steht die Toxizität $\left(\mathrm{LD}_{50}\right.$ : Maus und Ratte i.v. $3 \mathrm{mg} / \mathrm{kg}$, Maus und Ratte oral 32 bzw. 132 mg/kg, Ratte s. c. $41 \mathrm{mg} / \mathrm{kg}$ ) einer Verwendung dieses Esters im Wege.

\section{Andere Alkaloide}

Die folgenden drei Alkaloide, von denen zwei aus tierischen Organismen stammen, gehören verschiedenen Klassen an, besitzen aber als gemeinsames Strukturmerkmal eine oder mehrere Guanidingruppierungen.

Bei der Suche nach biologisch aktiven Substanzen aus marinen Organismen wurde aus dem methanolischen Gesamtextrakt der in Japan vorkommenden Koralle Parazoanthus gracilis und ihrem Wirt Dentitheca habereri, der Histaminähnlich wirkte, als basische Hauptkomponente auch eine Substanz mit Papaverin-ähnlicher Wirkung isoliert und Paragracin (XL) genannt [92]. Die Röntgenstrukturanalyse zeigte, daß die Verbindung das gleiche Grundgerüst besitzt wie die Pseudozoanthoxanthine [93], Pigmente aus dem Zoanthid Epizoanthus arenaceus, nämlich ein 1,3,7,9-Tetrazacyclopent $[\mathrm{e}] \mathrm{azulen}-\mathrm{System}$, und sich von diesen nur im Methylierungsmuster unterscheidet. Paragracin zeigte an aver-: schiedenen isolierten Organen antagonistische Wirkung bei Bariumchlorid-, Histamin-, Bradykinin- und Acetyl-cholininduzierten Spasmen in der Art des Papaverins.

Saxitoxin (XLI) ist ein Neurotoxin und eine der am meisten toxischen niedermolekularen Verbindungen, die wir kennen ( $\mathrm{LD}_{50}$ Maus $5-10 \mu \mathrm{g} / \mathrm{kg}$ ). Es wurde aus 


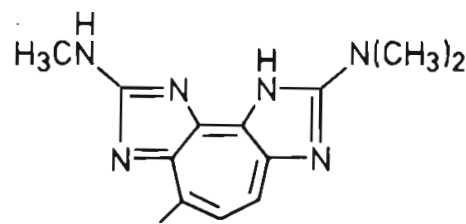

XL Paragracin

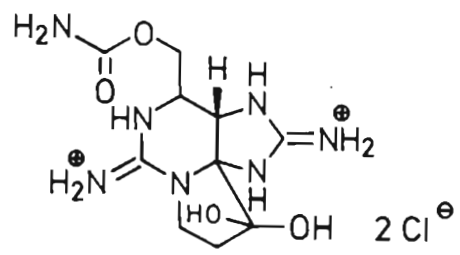

XLI Saxitoxin-hydrochlorid<smiles>COc1cc(C(=O)OCCCCNC(=N)N)cc(OC)c1O</smiles>

XLII Leonurin

Muscheln, und zwar aus den Verdauungsdrüsen von Mytilus californianus und aus den Syphons von Saxidomas giganteus, sowie aus den marinen Dinoflagellaten Gonyaulax catanella und $G$. tamarensis isoliert. Die Strukturaufklärung des hydrierten Pyrrolo-purins erfolgte auf der Basis von Röntgenstrukturanalysen. (Näheres zur Chemie s. in [17]). Saxitoxin blockiert die Nervenleitung in gleicher Weise wie Tetrodotoxin, d. h. durch selektive Blockade des $\mathrm{Na}$-IonenTunnels. Diese Eigenschaft allein würde aber die Verbindung kaum für unser Thema interessant machen. Kürzlich wurde jedoch seine interessante Wirkung im Zusammenhang mit Lokalanästhetika entdeckt: Trotz der geringen Lipidlöslichkeit werden in Gegenwart von Vasokonstriktoren seine lokalanästhetische Wirkung verstärkt und seine systemische Toxizität verringert [94]. Bei gleichzeitiger Gabe von Saxitoxin, eines Vasokonstriktors und eines Lokalanästhetikums bleiben systemische toxische Effekte aus, und man beobachtet ein rasches Einsetzen der Blockade, wie er für das Lokalanästhetikum charakteristisch ist, gekoppelt mit der langen Wirkungsdauer von Saxitoxin. Außerdem zeigen Lokalanästhetika bei Kombinationen mit kleinsten Mengen Saxitoxin einen Synergismus. Solche Kombinationen könnten nützlich sein, wenn eine lange Wirkungsdauer gewünscht wird [95].

Leonurus artemisia (Lamiaceae) wird in der chinesischen Volksmedizin unter dem Namen „I-mu Ts'ao“ seit Jahrhunderten bei gynäkologischen Erkrankungen und in der Geburtshilfe angewendet. Kritische Durchsicht der Literatur über diese Droge ließ erkennen, daß ihr therapeutischer Wert auf einem uterotonen Prinzip, das in den Blättern der blühenden Pflanze lokalisiert ist, beruht. Aus diesen wurde Leonurin isoliert, dessen Struktur mehrmals revidiert und schließlich als Syringinsäure-4-guanidinobutylester (XLII) erkannt wurde [96], wie 
auch durch verschiedene Synthesen ausgehend von Syringinsäure sichergestellt werden konnte [97]. Der uterotone Effekt wurde kürzlich mit Hilfe isometrischer Studien von Kontraktionen des Rattenuterus in vitro demonstriert [96]: Bereits ab $0,4 \mu \mathrm{g} / \mathrm{ml}$ erfolgten reguläre Kontraktionen großer Amplitude. Beim nicht kontrahierenden Uterus setzten diese nach einer Latenzzeit von mindestens 30 Minuten ein; der bereits kontrahierende Uterus reagierte mit erhöhter Kontraktionsrate [98].

\section{Terpenoide}

Diese Substanzklasse ist dabei, den Alkaloiden als den "klassischen“ Naturstoffen nicht nur durch die Anzahl ihrer neu aufgefundenen Vertreter sondern auch in pharmakognostischer, struktureller und biologischer Hinsicht etwas den Rang streitig zu machen.

\section{Mono- und Sesquiterpene}

1975 erschien eine Arbeit von DF.1. $C_{A}$. stulo et al. mit dem Titel „Marihuana, Absinth und das ZNS“, worin auf die auffälligen Ähnlichkeiten zwischen den psychischen Wirkungen des Likörs Absinth und den Erfahrungen von Marihuanarauchern hingewiesen wurde [99]. Thujon und $\triangle^{9}$-Tetrahydrocannabinol $\left(\triangle^{9}-\mathrm{THC}\right)$, die man für die aktiven Prinzipien von Artemisia absinthium bzw. Cannabis sativa hält, sind Terpenoide ähnlichen biogenetischen Ursprungs und besitzen ähnliche molekulare Geometrie, wenn man für Thujon als aktive Form das Enol annimmt (s. Formeln XLIII und XLIV, in denen gemeinsame Bin-

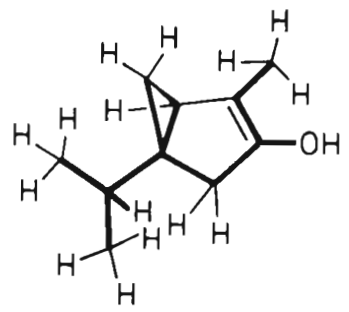

XLIII Thujon-enol
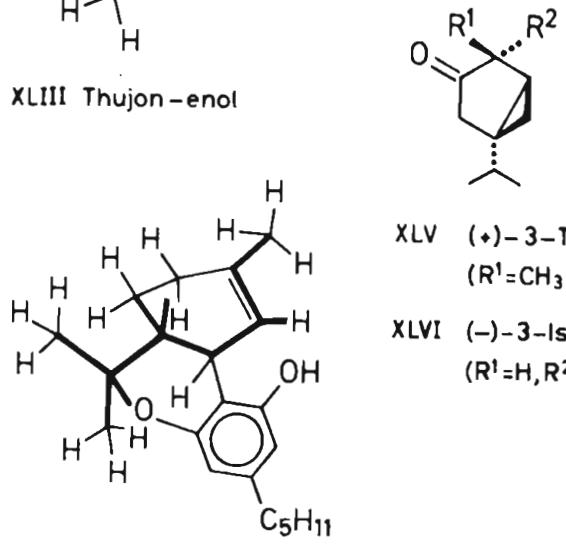

$X L V \quad(+)-3-T h u j o n$

$\left(\mathrm{R}^{\prime}=\mathrm{CH}_{3}, \mathrm{R}^{2}=\mathrm{H}\right)$

XLVI (-)-3-Isothujon

$\left(\mathrm{R}^{1}=\mathrm{H}, \mathrm{R}^{2}=\mathrm{CH}_{3}\right.$ )

XLIV $\Delta^{9}-T H C$ 
dungen dick ausgezogen sind). Nach eingehenden Betrachtungen über aktive Metaboliten des $\triangle^{9}$-THC und des Thujon, stellten die Autoren die Hypothese eines gemeinsamen Rezeptors im ZNS für beide Substanzen auf und schlugen genauere pharmakologische und toxikologische Untersuchungen mit dem noch wenig erforschten Thujon vor. Noch im gleichen Jahr untersuchte eine Gruppe am National Institute of Health (Bethesda, USA) die auf der Suche nach neuen nicht-suchterzeugenden Analgetika die Tetrahydrocannabinole, deren Metaboliten und eine große Zahl synthetischer Analoga eingehend erforscht hatte, (+)3-Thujon (XLV) und seinen Antipoden, das (-)-3-Isothujon (XLVI), die beide im ätherischen $\mathrm{Ol}$ von Artemisia-Arten (Compositae) vorkommen [100]. Während Thujon analgetisch unwirksam war, zeigte Isothujon im Heizplatten-Test eine mit Codein vergleichbare Wirkung
$\left(\mathrm{ED}_{50}\right.$ Maus s.c. $\left.6,5 \mathrm{mg} / \mathrm{kg}\right)$. Die Frage nach einem gemeinsamen Rezeptor von Thujon und THC blieb allerdings bei diesen Studien offen. Ebensowenig zeigte eine Reihe synthetischer Thujon-Analoga einen Ansatzpunkt für weitere Studien in analgetischer Richtung. Interessant ist aber die relativ starke analgetische Wirkung des kleinen $\mathrm{N}$-freien Moleküls 3-Isothujon auf jeden Fall.

Aus anderen Artemisia-Arten (Compositae) wurden zu Beginn der 60er Jahre zwei Sesquiterpenlactone vom SelinanTyp isoliert: Vulgarin aus Artemisia canariensis [101] und Tauremesin aus $A$. taurica [102]. Im Zuge der Strukturaufklärung erwies sich die Identität beider Verbindungen (XLVII). 1967 wurde über die hypoglykämische Wirkung dieses Terpens berichtet [103]: An Ratten erfolgte mit $0,5-1 \mathrm{mg} /$ Tier p.o. innerhalb 10 Minuten ein Glucose-Abfall von $30 \%$ (das entspricht in etwa der Wir-<smiles>CC1C(=O)O[C@H]2C1CC[C@]1(C)C(=O)C=C[C@H](O)[C@@H]21</smiles>

XLVII Vulgarin $=$ Tauremisin<smiles>CC(C)C(=O)O[C@H]1C[C@H](C)C2=CC(=O)[C@@](C)(C[C@@H]1OC(=O)C(C)C)O2</smiles>

XLVIII
A<smiles>C=C(C)C(=O)O[C@H]1C[C@@]2(C)OC(O)(C[C@H]2O)C(C)=C[C@@H]1OC(=O)C(C)C</smiles>

$X L I X$ 
kung von Insulin oder Tolbutamid); zusätzlich wurde bei i.v.-Gabe von $1 \mathrm{mg}$ eine hypolipämische Wirkung beobachtet. Clber die Digitalis-ähnlichen und parasympathomimetischen Effekte dieses auch unter dem Namen Judaicin aus $A r-$ temisia judaica isolierten Terpens wurde bereits früher berichtet (zitiert in [15], S. 430).

Im Rahmen eines Programms zur Isolierung von Sesquiterpenen aus Compositen wurden aus dem in Mexiko beheimateten Strauch Zexmennia brevifolia zwei Germacranolide, Zexbrevin A und $B$ (XLVIII, XLIX), isoliert und strukturell aufgeklärt [104]. Wurden Mäuse vor einer einzelnen Immunisierungsdosis von Schaf-Erythrozyten, Rinderserum oder Ei-Albumin mit $\mu \mathrm{g}$-Dosen der Zexbrevine behandelt, so wiesen sie eine höhere Zahl von spezifisch rosettenförmigen Zellen in der Milz wie auch höhere Antikörper-Titer auf als die nur mit Antigenen stimulierten Tiere. $500 \mu \mathrm{g} / \mathrm{Tier}$ war die optimale Dosis für den Anstieg rosettenförmiger Zellen. Die Zexbrevine mußten dazu $48 \mathrm{~h}$ vor Antigen-Stimulierung verabreicht werden; kein Effekt trat bei Gabe 3-11 Tage vor der oder am gleichen Tag der Antigengabe auf. In Gegenwart der Zexbrevine erfolgte kein Anstieg der Aufnahme radioaktiven Thymidins in einer Kultur von Maus-Milz-Zellen, was einen mitogenen Effekt ausschließt. Die Zexbrevine bewirken also bei der Maus eine starke Stimulierung der Immun-Antwort [105].

Am Central Drug Research Institute in Lucknow (Indien) wurde während des biologischen Screenings indischer Pflanzen beobachtet, daß ein $50 \%$ iger äthanolischer Extrakt aus dem Holz der Himalaya-Zeder Cedrus deodara (Pinaceae) deutliche spasmolytische Aktivität besaß. Als spasmolytische Hauptkomponente konnte das schon seit 1968 bekannte Sesquiterpen Himachalol (L) identifiziert werden [106]. Weitere aktive, in geringerer Menge vorkommende Terpene sind Allohimachalol (LI) [106] und das erst kürzlich strukturell aufgeklärte Centdarol (LII) [107]. Von diesen drei bicyclischen Sesquiterpenen besitzen der ungesättigte Alkohol Himachalol und das Diol Centdarol das gleiche Grundgerüst, Himachalon genannt, während sich Allohimachalol von einem isomeren Kohlenwasserstoff ableitet. Diese Verbindungen sind die ersten bekannten spasmolytisch hochwirksamen Sesquiterpene (s. a. [2], S. 217). Alle drei besitzen in vitro eine unspezifische spasmolytische Aktivität ähnlich der des Papaverins (Modell: isoliertes MeerschweinchenIleum, durch verschiedene Agentien induzierte Spasmen), wobei Allohimachalol um den Faktor 2-4 stärker als Himachalol und dieses etwa doppelt so stark wie Papaverin wirkt; Centdarol ist in vitro um den Faktor 3-5 schwächer als Pa-

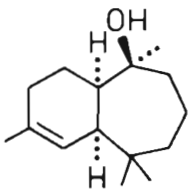

L Himachalol

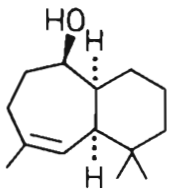

LI Allohimachalol

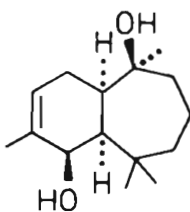

LII Centdarol 
paverin, in vivo (Katze, intraduodenal, Carbachol-Spasmen) zeigt Centdarol den ausgeprägtesten Effekt $[108,109]$.

\section{Diterpene}

Ebenfalls im Central Drug Research Institute in Lucknow (Indien) wurde 1977 aus den Wurzeln von Coleus forskoblii (Labiatae), deren Extrakt als hypotensiv und spasmolytisch wirkend bekannt war, ein Diterpen vom I.abdanTyp isoliert, das Coleonol genannt und u. a. durch Röntgenstrukturanalyse aufgeklärt wurde [110]. Im gleichen Jahr wurde von Hoechst in Deutschland und Indien aus der gleichen Pflanze ein Diterpen isoliert und Forskolin genannt [111]. Hoechst behauptet, die beiden Diterpene seien identisch (LIII) (Röntgenstruktur-

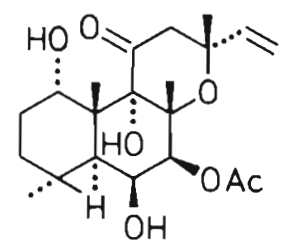

LIII Forskolin $=$ Coleonol

analyse liegt auch von einem ForskolinDerivat vor), während in der Publikation aus Lucknow die umgekehrte sterische Anordnung der Acetoxygruppe angegeben ist. Hoechst hat eingehend über die Pharmakologie des Forskolins berichtet [112]: Die Substanz übt auf das isolierte Meerschweinchen-Herz sowie auf das Hunde- und Katzen-Herz in situ eine ausgeprägte positiv inotrope Wirkung aus und steigert die Herzfrequenz. Beide Wirkungen werden durch B-Blocker nicht gehemmt. Auch an reserpinierten Hunden sind die Herz-Wirkungen nachweisbar. Die positiv inotrope Wirkung unterscheidet sich von der des Theophyllins, der Herzglykoside und des Veratrins. Forskolin senkt außerdem den Blutdruck bei Hunden und Katzen sowie bei spontan und renal hypertonen Ratten (mit $10 \mathrm{mg} / \mathrm{kg}$ sogar länger als $24 \mathrm{~h}$ ). (Eine Blutdrucksenkung von Coleonol an der Katze war bereits früher beobachtet worden, s. [20], S. 218.) Substanzen wie das Forskolin, die positiv inotrop wirken, sich aber in ihrem Wirkungsmechanismus von den bekannten positiv inotropen Substanzen, wie Catecholaminen, Herzglykosiden, Xanthinen oder Ceveratrum-Alkaloiden, unterscheiden, sind von großem theoretischen und praktischen Interesse; ebenso ist die blutdrucksenkende Aktivität dieser neuen chemischen Struktur auffallend.

Innerhalb eines Programms zur Erforschung von Pflanzeninhaltsstoffen mit Antiulcus-Wirkung stieß man in der japanischen Firma Sankyo auf "Plau-noi“, eine Droge, die in Thailand volksmedizinisch als Anthelmintikum und Dermatologikum Verwendung findet, und bei der es sich um den Stamm von Croton sublyratus (Euphorbiaceae) handelt. Ein Aceton-Extrakt dieser Droge inhibierte Shay-Ulcera bei Ratten und Reserpin-induzierte Ulcerationen bei Mäusen signifikant. Die Auftrennung des Extrakts erfolgte durch Verteilung zwischen n-Hexan und $80 \%$ igem wässerigem Methanol, wonach der Benzol-lösliche Anteil der Methanol-Phase die pharmakologische Aktivität besaß. Nach Entfernung inaktiver saurer Substanzen wurden aus dem Äther-löslichen Teil des BenzolRückstands, der Reserpin-Ulcera inhibierte, ein cyclischer Diterpen-Alkohol, aus dem Ather unlöslichen Anteil, der gegen Shay-Ulcera aktiv war, zwei Fura- 


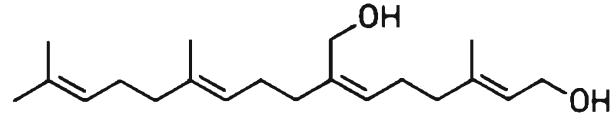

LIV 18-Hydroxy-geranylgeraniol

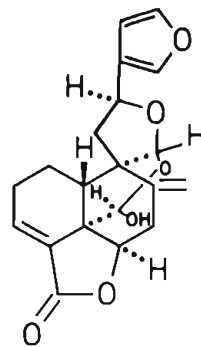

LV

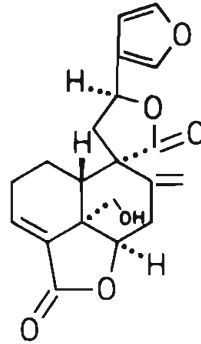

LVI B

Plaunol

no-diterpene isoliert. Das acyclische Diterpen konnte durch Vergleich seiner Spektren mit denen bekannter Diterpene und eine stereospezifische Synthese als 18-Hydroxy-geranylgeraniol (= E,Z,E)7-Hydroxymethyl-3,11,15-trimethyl2,6,10,14-hexadecatetraen-1-ol) identifiziert werden [113]. Die Verbindung besitzt bemerkenswerte inhibitorische Aktivität bei verschiedenen experimentell erzeugten Ulcerationen $\left(\mathrm{ID}_{50}{ }^{-}\right.$ Werte: Reserpin-Ulcus (Maus i.p.) < 10 mg/kg; Streß-Ulcus (p.o.) Maus 195, Ratte $73 \mathrm{mg} / \mathrm{kg}$; Aspirin-Ulcus (Ratte p.o.) $190 \mathrm{mg} / \mathrm{kg}$; zum Vergleich: Gefarnat in allen Modellen um $300 \mathrm{mg} /$ $\mathrm{kg}$ ). Die beiden Furano-diterpenlactone wurden Plaunol $A$ und $B$ genannt [114]. Die Struktur des Plaunols A wurde durch Röntgenstrukturanalyse seines $\mathrm{p}$ Brombenzoats als LV, die des strukturell ähnlichen, aber keine Acetal- und Halbacetal-Gruppierung enthaltenden Plaunols B aus den spektralen Daten und der chemischen Korrelation mit Plaunol A als LVI bestimmt. Plaunol B inhibierte mit i.p.-Gaben von 3 und $10 \mathrm{mg} / \mathrm{kg}$ Shay-Ulcerationen an der Ratte zu 55 bzw. $85 \%$; Plaunol A war in diesem Modell wirkungslos.

Schließlich sei noch ein Diterpen mit Steroid-ähnlicher Struktur vorgestellt: 11-Desacetoxy-wortmannin ist ein neuer Metabolit aus dem Schimmelpilz Penicillium funiculosum, der bei der Firma Sandoz isoliert wurde [115]. Seine Struktur (I.VII) basiert auf spektralen Daten und der Korrelation mit Wortmannin (LVIII), das schon früher aus Penicillium wortmanni isoliert worden war. Es handelt sich um 4-Methylsteroide, in denen der Ring A durch Spaltung und anschließende Lactonisierung modifiziert ist. Beide Verbindungen wirken schwach antimykotisch. 11-Desacetoxywortmannin ist außerdem antiinflammatorisch hoch wirksam, und zwar hat es einen starken Effekt auf akute Odeme wie auch auf 
entzündliche Gewebeproliferationen [116]. Seine Aktivität bei oraler Gabe im Carrageenin-Odem-Test ist ca. $1 / 4$ der von Dexamethason, gleich der von Indomethacin und $6 \mathrm{mal}$ größer als die von Phenylbutazon. Ahnliche Ergebnisse bei bilateral adrenalektomierten Ratten zeigen, daß für die Wirkung dieser Substanz eine Stimulierung der Nebennieren zumindest von untergeordneter Bedeutung ist. Im Granulombeutel-Test ist Desacetoxywortmannin weniger aktiv als Dexamethason, aber gleich gut oder besser als die nicht-steroidalen Antiphlogistika. Im Adjuvans-Arthritis-Test hat es keinen Effekt auf die primäre Schwellung, reduziert aber die Symptome einer generalisierten Arthritis dosisabhängig. Diese interessanten Eigenschaften des Desacetoxy-wortmannins sowie seine Steroidähnliche Struktur veranlaßten die Sandoz-Chemiker zur Synthese von Hydrocortison-ähnlichen Derivaten dieser Verbindung sowie des Wortmannins, indem sie in 17-Stellung beider Verbindungen die Corticoid-Seitenkette einführten (LIX, LX) [117] und die Acetoxygruppe des Wortmannins in eine Hydroxy- oder Carbonyl-Funktion umwandelten [118]. Während die antiinflammatorische Wirkung bei Veränderung der 11-Funktion deutlich abgeschwächt wurde, ging sie bei Einführung der Corticoid-Seitenkette vollständig verloren [116].

\section{Andere Terpenoide}

In der Ayurveda-Literatur wird „Guggulu“, das Harz aus Commiphora mukul (syn. Balsamodendron mukul, Burseraceae), als wirksam bei rheumatoider Arthritis, Fettleibigkeit und verwandten Krankheiten beschrieben. In den vergangenen 15 Jahren wurden in pharmakologischen und klinischen Studien mit dem Harz und einigen seiner Fraktionen antiinflammatorische und hypolipämische Aktivitäten der Droge erkannt [119]. Das Harz enthält Steroide, Diterpene, aliphatische Ester und Kohlenhydrate. Aus der Petroläther-Fraktion wurden u. a. zwei $\mathrm{C}_{21}$-Steroide, E- und Z-Guggulsteron (LXI, LXII), das cis- und trans-Isomere des 4,17(20)-Pregnadien-3,16-dions, isoliert [120]. Die hypolipämische Aktivität beider Steroide, bestimmt durch in vitro und in vivo Untersuchungen zur Lipolyse und Cholesterinbiosynthese, soll mit der von Clofibrat vergleichbar sein $[119,121]$. Zusätzlich zeigten $\mathrm{E}$ - und ZGuggulsteron sowie ihre Mischung in einer Endkonzentration von $10^{-4}$ molar auf die durch ADP, Adrenalin oder Seroto-

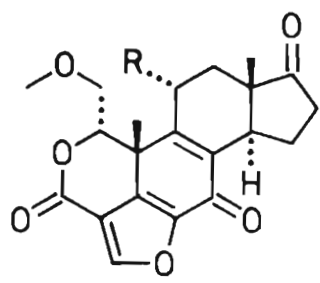

LVII 11-Desacetoxy wortmannin $(R=H)$

LVIII Wortmannin ( $R=O A C)$

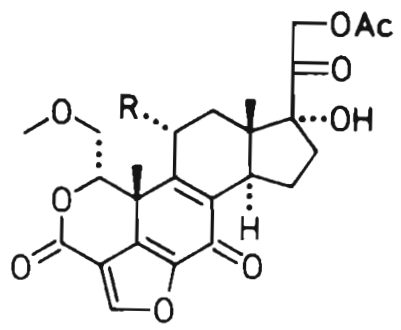

LIX $\mathrm{R}=\mathrm{H}$

LX $R=O A C$ 
nin induzierte Thrombocytenaggregation einen sehr ähnlichen Hemmeffekt wie Clofibrat [122].

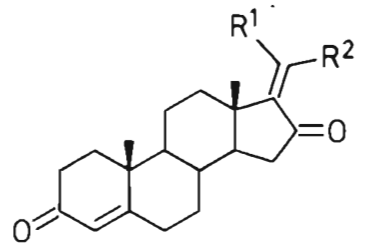

$\begin{array}{lll}\text { LXI } & E-\text { Guggulsteron } & \left(R^{1}=\mathrm{CH}_{3}, \mathrm{R}^{2}=\mathrm{H}\right) \\ \text { LXII } & Z- & \left(R^{1}=\mathrm{H}, \mathrm{R}^{2}=\mathrm{CH}_{3}\right)\end{array}$<smiles>Cc1cc2c(C(C)C)c(O)c(O)c(C=O)c2c(O)c2c3c(C(C)C)c(O)c(O)c(C=O)c3c(O)c1-2</smiles>

LXIII Gossypol

Das vor allem in Samen, aber auch in Wurzeln und subepidermalen Blattdrüsen von Gossypium-Arten (Malvaceae), zu denen auch die Baumwollpflanzen gehören, vorkommende gelbe Pigment Gossypol (LXIII) ist lange bekannt und wird u. a. als Antioxidans in der Gummiindustrie verwendet. Trotz seiner für Naturstoffe ungewöhnlichen 2,2'-Binaphthyl-Struktur ist es als dimeres Sesquiterpen $\left(\mathrm{C}_{30}\right)$ aufzufassen, wie auch biogenetische Studien zeigten. Seine chemischen, stereochemischen (Atropisomerie) und toxikologischen Aspekte sind in Ubersichtsartikeln behandelt [123125]. In den fünfziger Jahren fanden chinesische Forscher, daß die Verwendung von rohem Baumwollsamenöl (Oleum Gossypii) zum Kochen zur Infertilität führen kann. Untersuchungen in den sechziger Jahren zeigten dann, daß der männliche Organismus bezüglich der antifertilen Effekte empfindlicher reagiert als der weibliche. Schließlich wurde an Tieren demonstriert, daß Gossypol für die antifertile Wirkung des Ols verantwortlich war. Nach 2- bis 4wöchiger oraler Gossypol-Gabe von 15-40 mg/ $\mathrm{kg} / \mathrm{Tag}$ wurden männliche Ratten infertil, wobei der Wirkungseintritt dosisabhängig zu sein schien. Die Wirkung hielt noch 3-5 Wochen nach Absetzen des Gossypols an; danach regenerierte sich die Fertilität allmählich. Ab 1972 wurden in der Volksrepublik China (National Coordinatory Group on Male Antifertility) über 4.000 männliche Versuchspersonen über 6 Monate mit täglich $20 \mathrm{mg}$ Gossypol behandelt. Durch EjakulatUntersuchungen ließ sich zeigen, daß die Infertilität gewöhnlich erst nach zwei Monaten vollständig war. Der Erfolg (Nekrospermie oder Spermienzahl unter 4 Millionen/ml) lag bei $99,89 \%$. Nach Beginn der Sterilität genügte eine Erhaltungsdosis von $150-220 \mathrm{mg} /$ Monat (in mehreren Einzeldosen). An Nebenwirkungen beobachtete man anfängliche, aber vorübergehende Schwäche, ferner Appetit-Veränderungen, epigastrische Störungen sowie einen gewissen LibidoVerlust ohne Beeinträchtigung der Potenz [126]. Zur Zeit laufen in China Versuche mit Gossypol an ca. 10.000 freiwilligen männlichen Versuchspersonen [127].

\section{Sauerstoff-Heterocyclen}

In dieser Gruppe, zu der biogenetisch so unterschiedliche Naturstoffe wie einige Phenylpropanabkömmlinge (z. B. Cumarine) und Xanthone genauso gehö- 
ren wie viele Polyketide (z. B. Phenylchroman-Derivate vom Typ der Flavane, Iso- und Neoflavane), werden nach wie vor viele neue Vertreter in der Natur gefunden. Allerdings weichen sie in ihren Strukturen und/oder ihren biologischen Eigenschaften meist wenig von bereits länger bekannten Vertretern $a b$, so daß hier nur vier Substanzen vorgestellt werden sollen, zumal noch einige weitere im nächsten Kapitel, bei den Glykosiden, behandelt werden.

Das Cumarin Scoparon (LXIV), der Aesculetin-dimethyläther, ist lange Zeit bekannt und auch synthetisch leicht zugänglich [128]. Es wurde u. a. aus den Blütenköpfen und Samen von Artemisia scoparia (Compositae) [129] und aus dem Kernholz von Fagara macrophylla (Rutaceae) isoliert [130]. Die ausgeprägte hypotensive Wirkung des Scoparons $[131,132]$ wurde an anästhesierten normotonen Tieren (Katze, Hund, Ratte) und an hypertonen Hunden demonstriert; sie ist dosisabhängig und setzt wenige Sekunden nach Applikation der Substanz (i.v. oder intraduodenal) ein. Mit $10 \mathrm{mg} / \mathrm{kg}$ senkt Scoparon beim Hund den Blutdruck stärker (58\%) und für längere Zeit $\left(160^{\circ}\right)$ als $\alpha$-Methyldopa (12\%, 120'). Die LD Lo -Werte betragen (in $\mathrm{mg} / \mathrm{kg}$ ): Ratte 292 (p.o:) bzw. 190 (i.p.), Maus 280 (p.o.) bzw. 225 (i.p.). Die Wirkung beruht weder auf einem cholinergen Mechanismus noch einer Histaminfreisetzung. Vielmehr erzeugt Scoparon eine periphere Vasodilatation, wirkt spasmolytisch und relaxierend an der glatten Muskulatur und außerdem milde sedierend und antikonvulsiv. In Indien ist Scoparon für klinische Tests freigegeben [133]. Aufgrund der interessanten Wirkung dieser einfachen Verbin- dung lag natürlich die Testung verschiedener andersartig substituierter Cumarine nahe. Ein entsprechendes Programm an einem indischen Forschungsinstitut zeigte, daß das optimale Substitutionsmuster offenbar durch Scoparon selbst repräsentiert wird. Das unsubstituierte Cumarin zeigt zwar ebenso eine gewisse hypotensive Wirkung wie das 5,7- und das 6,7-Dihydroxy-Cumarin (Aesculetin), aber Methylierung bewirkt beim ersten eine Abschwächung, beim letzteren eine Verstärkung der Wirkung [132].

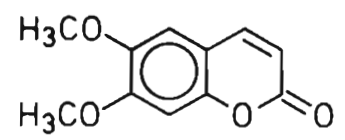

\section{Scoparon}

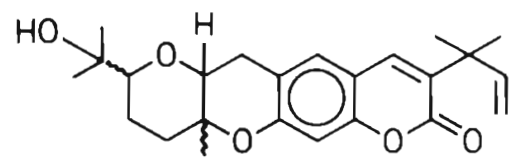

LXV, LXVI Clausmarine A und B

Am Central Drug Research Institute in Lucknow fand man in der Rutacee Clausena pentaphylla zwei spasmolytisch wirksame isomere Terpen-Cumarine, die man Clausmarin $A$ und $B$ (LXV, LXVI) nannte [134]. Wie aus spektroskopischen Daten (insbesondere ${ }^{13} \mathrm{C}$-Kernresonanz) und aus biogenetischen Uberlegungen abgeleitet wurde, handelt es sich um $\mathrm{Cu}$ marine, die in 3-Stellung mit einer Prenyl-Einheit substituiert und in 6,7-Stellung mit einem bicyclischen Monoterpen-Teil kondensiert sind. Die Stereochemie der Ringverknüpfung im Monoterpen-Teil ist bei beiden Clausmarinen offenbar gleich aber unbekannt; sie unterscheiden sich aber in der sterischen 
Anordnung der Isopropylgruppe. Über die spasmolytische Wirkung der Clausmarine wird nur gesagt, daß sie in verschiedenen Tiermodellen ausgeprägt ist. Trotz des Fehlens näherer pharmakologischer Einzelheiten sollten die Substanzen aber wegen ihrer neuartigen Struktur vorgestellt werden.

Die Fruchtschalen von Garcinia mangostana (Guttiferae) wirken adstringierend und werden in Indien bei Diarrhoe und Dysenterie angewendet. Aus diesem Pflanzenteil war bereits 1855 erstmals das Xanthon Mangostin isoliert, aber erst über ein Jahrhundert später endgültig in seiner Struktur (LXVII) aufgeklärt worden [135]. Wie die meisten Xanthone und ihre Glykoside wurde es bisher kaum pharmakologisch untersucht [136] (Ausnahme: Mangiferin, s. [1]). Eine indische Arbeitsgruppe bemühte sich jüngst um das pharmakologische Profil von Mangostin und einigen seiner partialsynthetischen Derivate, u. a. seinem Triacetat (LXVIII) und 1-

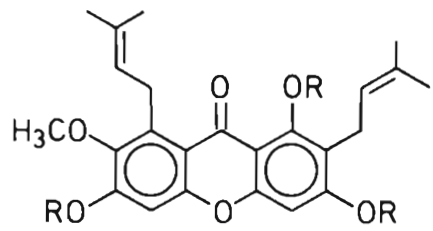

LXVII Mangostin ( $\mathrm{R}=\mathrm{H}$ )

LXVIII Mangostin-triacetat $(R=A C)$

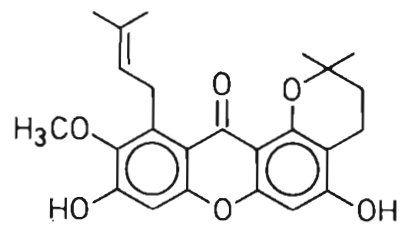

LXIX 1-Isomangostin
Isomangostin (LXIX) [137]. Alle drei Verbindungen zeigten bei Mäusen und Ratten eine ZNS-Depression, charakterisiert durch Ptosis, Sedierung, verminderte motorische Aktivität, Potenzierung der Pentobarbital-Schlafzeit und derÄther-Narkose, aber keine analgetischen, antipyretischen oder antikonvulsiven Effekte. Außerdem sind die Verbindungen an Ratten bei oraler und intraperitonealer. Verabreichung in verschiedenen Modellen (CarrageeninOdem, Baumwollkügelchen, Granulombeutel) antiinflammatorisch aktiv: in allen Modellen wirken die Xanthone mit $50 \mathrm{mg} / \mathrm{kg}$ etwa gleich stark wie $100 \mathrm{mg} /$ $\mathrm{kg}$ Phenylbutazon oder $1 \mathrm{mg} / \mathrm{kg}$ Dexamethason. Die Wirkung wird sogar an bilateral adrenalektomierten Tieren beobachtet. Im Gegensatz zu den nichtsteroidalen Antiphlogistika besitzt Mangostin (und nur dieses) in der angegebenen Dosierung einen signifikanten Anti-Ulcer-Effekt.

Im Rahmen eines Programms, dessen Ziel die Auffindung spezifischer, durch Mikroorganismen produzierter Inhibitoren der Cholesterol-Biosynthese war, isolierte man in der japanischen Firma Sankyo aus Penicillium citrinum drei Metaboliten: $M L-236 A, M L-236 B$ und $M L-236 C$ (LXX-LXXII) [138]. Kurz zuvor war das mit ML-236B identische Compactin (LXXII) in der englischen Firma Beecham als fungizider Metabolit in Kulturen von Penicillium brevicompactum entdeckt worden [139]. Die Strukturaufklärung der Verbindungen erfolgte in beiden Arbeitskreisen unabhängig voneinander durch Kombination spektroskopischer, chemischer und Röntgenstrukturanalytischer Methoden. Der Grundkörper wird wahrscheinlich 
durch Polyketid-Cyclisierung gebildet, wobei sowohl die Faltung der Nonaketid-Kette als auch die niedrige Oxidationsstufe für cyclische Polyketide ungewöhnlich sind. An erwachsenen Ratten,

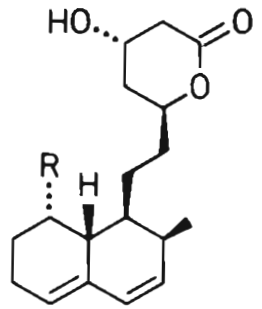

$$
\begin{array}{lll}
\text { LXX } & \mathrm{ML}-236 & \mathrm{C}(\mathrm{R}=\mathrm{H}) \\
\text { LXXI } & \mathrm{ML}-236 & A(\mathrm{R}=\mathrm{OH}) \\
\text { LXXII } & \mathrm{ML}-236 & \mathrm{~B}=\mathrm{Compactin} \\
& \left(\mathrm{R}=\mathrm{OC}(\mathrm{O}) \mathrm{CH}\left(\mathrm{CH}_{3}\right) \mathrm{CH}_{2} \mathrm{CH}_{3}\right)
\end{array}
$$

Hennen, Hunden und Affen konnten mit Compactin in oralen Dosen zwischen 10 und $50 \mathrm{mg} / \mathrm{kg} / \mathrm{Tag}$ signifikante Senkungen des Serumcholesterin-Spiegels demonstriert werden [140]. Die $\mathrm{LD}_{50}$ der Verbindung lag bei der Maus (oral) über $2 \mathrm{~g} / \mathrm{kg}$. Die beiden strukturell verwandten Metaboliten ML-236A und ML-236C besaßen deutlich geringere hypocholesterinämische Aktivitäten. Die Cholesterin-Synthese wird durch Compactin in isolierten Lymphocyten und intestinaler Mucosa von gesunden Versuchspersonen und Patienten mit familiärer Hypercholesterinämie signifikant gehemmt [141]. In menschlichen Fibroblasten inhibiert die Verbindung die 3-Hydroxy-3-methylglutaryl-Coenzym-AReductase kompetitiv [142]. An RattenHepatocyten ließ sich zeigen, daß die Verbindung lediglich den Acetat-Einbau in 3-Hydroxysteroide, nicht dagegen in andere Lipide hemmt [143].

\section{Glykoside}

Bei den für unser Thema relevanten Glykosiden handelt es sich um vier $\mathrm{O}$. Glykoside und zwei Nucleoside.

Im Naturstoff-Institut der Universität Laguna auf Teneriffa wurde 1976 aus Aeonium lindleyi, einer auf den kanarischen Inseln verbreiteten Crassulacee, das Glucosid Lindleyin isoliert [144]. Es erwies sich als ein phenolisches Gallylglucosid und zwar als 4-(4'-Hydroxyphenyl) - 2 - butanon-4'-O- $\beta-\mathrm{D}-\left(6^{\prime \prime}-\mathrm{O}-\right.$ gallyl)-glucopyranosid (LXXIII). Wegen

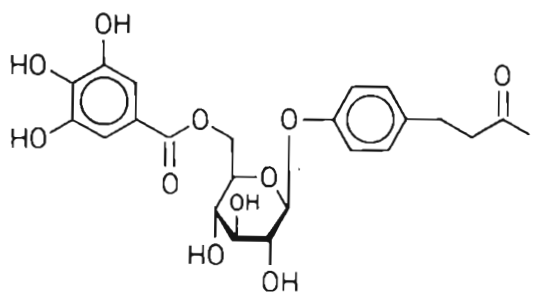

LXXIII Lindleyin

seiner gewissen biologischen Aktivität im Screening wurde es im gleichen Institut näher untersucht [145]. Im Siegmundund Randall-Sellito-Test besitzt Lindleyin eine dem Aspirin oder Phenylbutazon vergleichbare analgetische Wirkung, ist aber wirkungslos im Vergleich zu Morphin im Heizplattentest. Es ist antiinflammatorisch etwas aktiver als Aspirin im Carrageenin-Odem-Test und zwar sowohl bei normalen als auch adrenalektomierten Ratten. Ebenso ist es mit Aspirin in antiarthritischer Hinsicht (bei der durch Mycobacterium butyricum induzierten Polyarthritis an der Rattenpfote) vergleichbar, aber weniger antipyretisch wirksam. Ulcerationen traten nicht auf, aber eine Hypotension bei i.v.-Dosen über $25 \mathrm{mg} / \mathrm{kg}$. Die Toxizität der Ver- 
<smiles>COBOC1=C/C(=C\C(=O)O[GaH2])Oc2cc(O)cc(C)c21</smiles>

LXXIV

bindung ist gering ( $\mathrm{LD}_{50}$ Maus i.v. 395, i.p. 1260 , oral $2190 \mathrm{mg} / \mathrm{kg}$ ).

Aloe ist bekanntlich das aus den abgeschnittenen Blättern ausgeflossene, eingedickte und erstarrte Sekret verschiedener Aloe-Arten (Liliaceae), dessen Hauptbestandteil das Aloin, ein Anthron-C-glucosid, zu den sichersten und stärksten Laxantien zählt. Auch Aloe arborescens, das in der japanischen Volksmedizin u. a. bei gastrointestinalen Störungen Verwendung findet, soll Aloe liefern. In Japan wurden aus dem Blatt-Saft dieser Aloe-Art auch ein neues Glucosid, Aloenin genannt, und $\mathrm{Mg}$-lactat isoliert [146]. Als Struktur für Aloenin war ursprünglich die eines Chromenol-glucosids (LXXIV) abgeleitet worden [147]. Die gleichen Autoren legten später als revidierte Struktur die eines Hydroxyphenyl-2-pyron-glucosids vor (LXXV), die durch Röntgenstrukturanalyse gesichert ist [148]. Es konnte gezeigt werden, daß die Biosynthese in der Reihenfolge Polyketid-Cyclisierung zum Phenylpyron, O-Methylierung, Glucosidierung abläuft [149]. An der Shay-Ratte reduziert Aloenin in einer oralen Gabe von $100 \mathrm{mg} / \mathrm{kg}$ die Magensaftsekretion um $23 \%$, noch besser - mit $34 \%$ - wirkt das sog. Aloeulcin, das gleichfalls in Aloe arborescens vorkommt und sich als $\mathrm{Mg}$-lactat erwies $[146,148]$.<smiles>COc1cc(O)c(Oc2cc(O)cc(C)c2-c2cc(OC)c(O)c(=O)o2)c(O)c1</smiles>

LXXV Aloenin

"Tu-Chung", botanisch Eucommia ulmoides (Eucommiaceae), ist eine der ältesten bekannten Arzneipflanzen, deren medizinischer Wert seit Jahrtausenden in China bekannt ist. Der Extrakt gilt als allgemeines Tonikum. "Tu-Chung“Rinden-Tee oder - Wein bewirken deutliche Besserung der Symptome bei Hypertonikern. In neuerer Zeit wurde die hypotensive Wirkung der Extrakte in Tierversuchen bestätigt. Sie ist offensichtlich das Ergebnis peripherer Vasodilatation (durch direkte Wirkung auf die vaskuläre glatte Muskulatur). Obwohl einige Untersuchungen über die Inhaltsstoffe der Droge vorlagen, fehlte bisher eine systematische Suche nach dem aktiven Prinzip. SIH und Mitarbeiter berichteten 1976 über die Identifizierung und Synthese von Pinoresinol-diglucosid (LXXVI) als

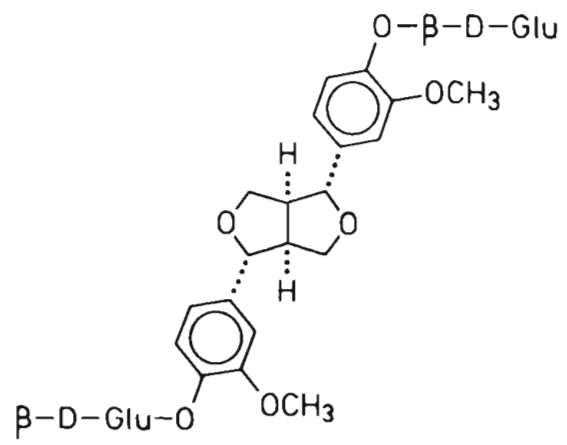

LXXVI Pinoresinol-diglucosid 
hypotensivem Prinzip der „Tu-Chung“Rinde [150]. Hydrolyse mit $\beta$-Glucosidase liefert zwei Mol Glucose und racemisches Pinoresinol, ein Zwischenprodukt bei der Biosynthese des Lignins aus Coniferylalkohol. Umgekehrt konnten die Autoren durch Umsetzung von synthetisch durch Dimerisierung von Coniferylalkohol in Gegenwart des Mikroorganismus Caldariomyces fumago gut zugänglichem Pinoresinol mit $\alpha$-Acetobromglucose Pinoresinol-diglucosid gewinnen, das in seinen physikalischen und biologischen Eigenschaften mit dem $\mathrm{Na}$ turprodukt identisch war. An spontan hypertensiven Ratten bewirkt die Verbindung durch die Jugularis appliziert einen Abfall des diastolischen Blutdrucks um 25-35 oder 80 oder $105-120 \mathrm{~mm} \mathrm{Hg}$ bei 30 bzw. 40 bzw. $100 \mathrm{mg} / \mathrm{kg}$.

Der alkoholische Extrakt von Breynia officinalis, einer in Taiwan gesammelten Euphorbiacee, zeigte bemerkenswerte hypocholesterinämische Wirkung. Die am Bristol-Banyu Research Institute in Japan erfolgte Fraktionierung und Gewinnung von zwei nahe verwandten aktiven Verbindungen, der Breynine $A$ und
$B$, wurde durch DC, UV-Messung (charakteristisches Maximum bei $258 \mathrm{~nm}$ ) und hypochlesterinämische Tests an Ratten verfolgt [151]. Weitgehend in seine Struktur aufgeklärt ist Breynin $\mathrm{A}$, ein $\mathrm{S} \frac{\mathrm{D}}{\mathrm{D}}$ haltiges Glykosid, das sich unter Abspal tung von Glucose und Rhamnose zunf Breynogenin (LXXVII) und weiter zung Breynolid (LXXVIII) und p-Hydroxy benzoesäure hydrolysieren läßt. Spek $\frac{{ }_{\omega}}{5}$ tren und chemische Reaktionen ergaben, daß es sich beim Breynolid um ein neuar tiges System handelt, nämlich um einene Tetrahydrofuran-Ring, der einerseits spiroartig unter Ausbildung einer Ketal gruppierung mit einem Tetrahydropy? ran-Ring verknüpft, andererseits mit ei nem Cyclohexan-Ring kondensiert ist, an den seinerseits ein Tetrahydrothio phen ankondensiert ist. Konstitution und Konformation dieses eigenartigen Systems wurden durch Röntgenstruktur $\frac{5}{\Phi}$ analyse gesichert. Das Breynolid enthäl neben einer Ketogruppe drei sekundäre und eine tertiäre Hydroxylfunktionen. Bei der im Breynogenin mit p-Hydroxy $-\frac{-}{\Phi}$ benzoesäure veresterten handelt es sich: um die im Tetrahydropyran-Ring; füro

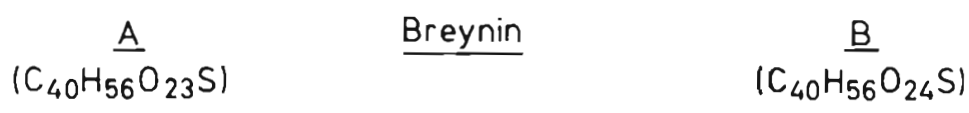

- Glucose

- Rhamnose

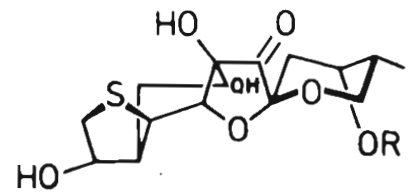

LXXVII

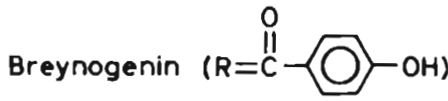

LXXVIII Breynolid $\quad(R=H)$ 
die Glykosidbindung konnte die tertiäre ausgeschlossen werden; eine oder beide der verbleibenden $\mathrm{OH}$-Gruppen können glykosidiert sein. Breynin B unterscheidet sich von Breynin A durch den Mehrgehalt eines Sauerstoffs; ob es sich um ein entsprechendes Sulfoxid oder um ein Hydroxy-breynin A handelt, ist noch offen [152]. $5 \mathrm{mcg} / \mathrm{kg} / \mathrm{Tag}$ Breynin A bzw. $25 \mathrm{mcg}$ Breynin $B$ bewirken an der Ratte (i.p.) einen Serumcholesterin-Abfall von 20 bzw. $32 \%$. An der Maus beträgt die $\mathrm{LD}_{50} 250 \mathrm{bzw} .410 \mathrm{mcg} / \mathrm{kg}$ [151].

Das Schicksal der meisten bisher isolierten Antibiotika, nämlich sich als praktisch wertlos zu erweisen, hätte auch das Bredinin geteilt, das eine gewisse, sehr enge Anticandida-, Antivirus- und Antitumor-Wirkung besitzt, wenn nicht pharmakologische Studien Hinweise auf seine immunsuppressiven Eigenschaften gegeben hätten [153]. Bredinin wurde bei der japanischen Firma Toyo Tozo aus Eupenicillium brefeldianum isoliert (aus diesem Pilz stammt auch das schon länger bekannte Brefeldin) [153]. Chemisch handelt es sich beim Bredinin um einen neuen Vertreter aus der Klasse der Imidazol-Nucleoside, nämlich um 4-Carbamoyl-1- $\beta$-D-ribofuranosyl - imidazolium-5-olat (LXXIX), wie Röntgenstrukturanalyse und Synthese bewiesen [155]. Seine immunsuppressiven Eigenschaften wurden zunächst an der SchafErythrozyten-Präparation bei Mäusen beobachtet; Bredinin ist dabei aktiver als Azothioprin, oral wirksam und inhibiert primäre und sekundäre Immunreaktionen [154]. Weil einige Immunsuppressiva beneficial side effects bei rheumatoider Arthritis und systemischem Lupus erythomatosus haben, wurde der Effekt von Bredinin auf die durch Mycobacterium butyricum induzierte Polyarthritis an der Rattenpfote untersucht; und zwar wurde das Nucleosid 30 Tage in einer Dosis von 2,5-10 mg/kg i.p. gegeben. Während die primäre Entzündungsphase innerhalb der ersten drei Tage unbeeinflußt blieb, erfolgte Suppression der Sekundärphase zwischen den 20. und 30. Tag und Ausbleiben sekundärer Läsionen um den 40. Tag (ähnliche Wirkung hat 6-Mercaptopurin). Bredinin ist relativ untoxisch $\left(\mathrm{LD}_{50}\right.$ Ratte: i.p. $>5 \mathrm{~g} / \mathrm{kg}$, i.v. $1,5 \mathrm{~g} / \mathrm{kg}$ ). Eine Dosis von $0,2 \mathrm{~g} / \mathrm{kg}$ über 6 Tage i.p. erniedrigt im Gegensatz zum Azothioprin die Leukozytenzahl nur unwesentlich [156]. Bredinin ist ein Beispiel für das Auffinden neuer oder ungewöhnlicher Wirkungen, die sich unter den antibakteriellen Eigenschaften aufgegebener Antibiotika verbergen.

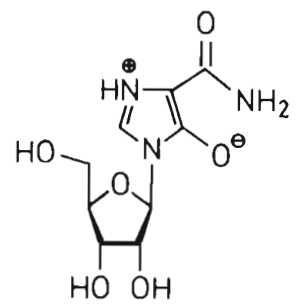

LXXIX Bredinin

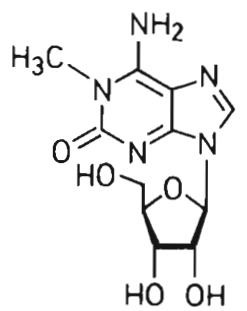

LXXX 1-Methyl-iso-guanosin 
Ein Patent der Firma Hoffmann-LaRoche (Basel) beschreibt ein neues $\mathrm{Nu}$ cleosid, 1-Methyl-iso-guanosin (= 1-Methyl-9קD-ribofuranosyl-iso-guanin) (LXXX) aus orangefarbenen Schwämmen der Gattung Tedania, die an der australischen Küste gesammelt wurden [157]. Die Isolierung erfolgte durch Extraktion der lyophilisierten Organismen mit polaren Lösungsmitteln und anschließende Kationenaustauscher-Chromatographie. Im gleichen Patent sind auch zwei Synthesen für die Verbindung aufgeführt: aus einem Imidazol-Derivat oder durch Methylierung von Iso-guano$\sin$. Neben verschiedenen pharmakologischen Aktivitäten (ZNS-Beeinflussung, antiinflammatorisch, antiallergisch, hypotensiv) soll 1-Methyl-iso-guanosin vor allem muskelrelaxierende Eigenschaften besitzen: Der $\mathrm{ED}_{50}$-Wert beträgt bei der Maus und intraperitonealer Applikation $3,1 \mathrm{mg} / \mathrm{kg}$ und bei oraler $12 \mathrm{mg} / \mathrm{kg}$. Am gleichen Tier ist seine $\mathrm{LD}_{50}$ nach 48 Stunden mit $1 \mathrm{~g} / \mathrm{kg}$ angegeben.

\section{Verbindungen mit anderen Strukturen}

In diesem Kapitel wurden Verbindungen zusammengefaßt, die sich strukturell und biogenetisch nicht in die vorherigen Kapitel einordnen ließen, und zwar einige Acetylenverbindungen und Aromaten.

Die Droge "Toki", das ist die Wurzel der Umbellifere Angelica acutiloba, wird heute in Japan kultiviert, nachdem sie aus der alten chinesischen Medizin als analgetisches, sedatives und antiinflammatorisches Mittel bekannt war. Ein wässeriger Extrakt soll im Writhing-Test an der Maus etwa 1,7mal wirksamer sein als
Acetylsalicylsäure, bezogen auf das Trockengewicht der Droge. Die Droge wurde an der Universität Kyoto auf antinozizeptive Bestandteile durch Fraktionierung und Biotests analysiert, wobei 7 Verbindungen erstmals aus "Toki“ isoliert wurden, die sich im pharmakologischen Test (durch Essigsäure induziertes "Writhing, von Mäusen) als wirksam herausstellten [158]: Falcarinol (LXXXI), Falcarindiol (LXXXII) [159], Falcarinolon (LXXXIII) $[160,161]$, Cholin, Scopoletin, Umbelliferon und Vanillinsäure. Die drei Polyacetylene erwiesen

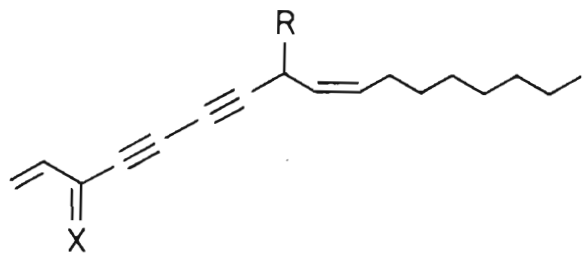

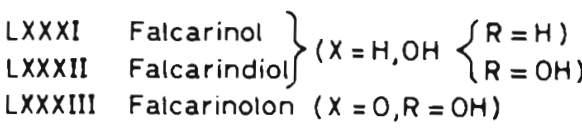

sich als am wirksamsten $\left(E D_{50}\right.$ per os: 0,3-0,4 mg/kg). Úberdies zeigte Falcarindiol die antinozizeptive Aktivität auch bei Injektion von Bradykinin in die Halsschlagader von Ratten (ED $\mathrm{ED}_{50}$ s.c. $22 \mathrm{mg} /$ $\mathrm{kg}$ ). Bei diesen Verbindungen handelt es sich um verwandte $\mathrm{C}_{17}$-Endiine mit unterschiedlichem Oxidationsgrad, die in den Arbeitskreisen von BOHLManN und E.R.H. Jones schon in den 60er Jahren aus anderen Umbelliferen isoliert worden waren. Für die große Zahl der natürlich vorkommenden Polyacetylene sind bisher nur antibakterielle oder antifungale Wirkungen beschrieben; insofern ist die gewisse analgetische Aktivität, insbesondere des Falcarindiols, neuartig und bemerkenswert. 
Als weitere Acetylenverbindungen seien einige Substanzen mariner Herkunft erwähnt, bei denen es sich um halogenierte cyclische Äther mit terminaler cisoder trans-En-in-Gruppierung in der Seitenkette handelt. Sie sind offenbar alle gleichen terpenoiden Ursprungs, wobei als Precursor für die in Gegenwart von Halogenen ablaufenden Cyclisierungen Pentadeca - 3,9,12-trien - 1 - in-6,7-diol postuliert wurde [162]. Dactylyn (LXXXIV), ein trishalogeniertes Tetrahydropyran-Derivat, wurde aus der Molluske Aplysia dactylomela, auch als Seehase bekannt, isoliert, stammt aber vermutlich aus Algen, die diesen Weichtieren als Nahrung dienen [163]. Die vier anderen, einen 8-Ring-Äther enthaltenden Verbindungen Laureatin (LXXXV), Isolaureatin (LXXXVI), Laurefucin (LXXXVII) und Laurencin (LXXXVIII) kommen in verschiedenen Rotalgen der Gattung Laurencia (Rhodomelaceae) vor $[164,162,165]$. Ihre Strukturaufklärung war verhältnismäßig schwierig und letzten Endes nur mit Hilfe der Röntgenstrukturanalyse möglich [166]. Für Laurencin wurde inzwischen auch eine Syn- these beschrieben [167]. Am Institut für Marine Pharmakologie der Universität von Oklahoma entdeckte man, daß alle diese fünf Substanzen den PentobarbitalMetabolismus inhibieren und damit die durch dieses Pharmakon induzierte Schlafzeit verlängern $[168,169]$. Dactylyn ist die wirksamste Substanz [170]: Eine i.p.- oder i.v.-Dosis von $25 \mathrm{mg} / \mathrm{kg}$ verlängert bei der Maus die Pentobarbital-Schlafzeit um mehr als 10 Stunden. Ein Hinweis auf die Inhibierung des Metabolismus war der Anstieg der Eliminierungs-Halbwertszeit von Pentobarbital in Dactylyn-behandelten Mäusen um das Mehrfache gegenüber unbehandelten Tieren. Dactylyn ist an der Maus bei i.v.Gabe bis $200 \mathrm{mg} / \mathrm{kg}$ untoxisch. Aus der Laurencia-Gruppe ist Isolaureatin die aktivste Substanz. Interessant ist, $\mathrm{da} ß$ keine der Verbindungen in den zur Metabolismus-Inhibierung notwendigen Dosierungen pharmakologische Eigenwirkungen zeigte. Diese Eigenschaft zusammen mit hoher Wirksamkeit bei der Metabolismus-Inhibierung und geringer Toxizität könnten diese Verbindungen klinisch interessant werden lassen.

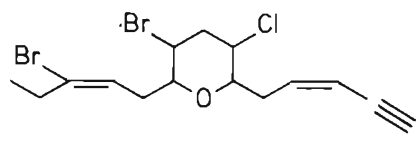

LXXXIV Dactylyn

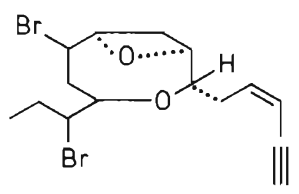

LXXXV Laureatin

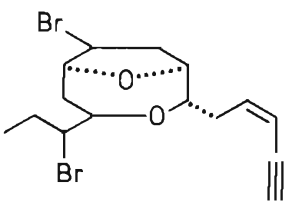

LXXXVI Isolaureatin

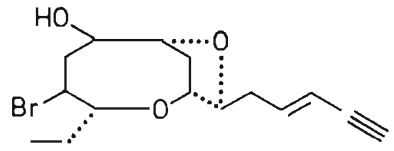

LXXXVII Laurefucin

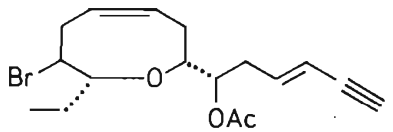

LXXXVIII Laurencin 
Der methanolische Extrakt aus den Samen von Alpinia galanga (Zingiberaceae), unter dem Namen "Houdoukou“ eines der Magenmittel in der chinesischen Medizin, zeigte signifikante Wirkung auf Ulcerationen an Shay-Ratten. Die Firma Sankyo berichtete kürzlich über die Isolierung, Strukturaufklärung und Synthese zweier in diesem Modell wirksamer $\quad \alpha$-Vinyl-benzylalkohole [171]. Es handelt sich um die offenbar noch nicht beschriebenen Verbindungen I'-Acetoxychavicol-acetat

(LXXXIX) und sein 3-Methoxy-Derivat 1'-Acetoxyeugenol-acetat (XC). An Shay-Ratten zeigten beide Substanzen in Dosen zwi-

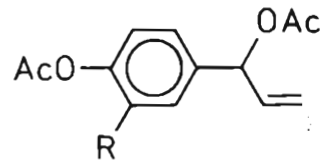

\section{1 -Acetoxychavicol-acetat $(\mathrm{R}=\mathrm{H})$ $X C \quad 1$-Acetoxyeugenoi-acetat $(R=O A C)$}

schen 2 und $10 \mathrm{mg} / \mathrm{kg}$ einen Anti-UlcerEffekt, während Acetoxy-chavicol-acetat mit $2 \mathrm{mg} / \mathrm{kg}$ auch signifikant die Magensaftsekretion hemmte. Dabei hatten das $S$-Konfiguration besitzende natürliche und das racemische synthetische Chavicol-Derivat praktisch den gleichen Effekt auf die Ulcera, während das racemische Eugenol-Derivat nur etwa die halbe Wirkungsstärke des natürlichen mit unbekannter Stereochemie besaß.

Ebenfalls aus der überlieferten chinesischen Medizin stammt die bei gastrointestinalen Beschwerden und beim Parkinson angewendete Droge „Houpo“, d. i. die Rinde von Magnolia officinalis (Magnoliaceae). Ein Extrakt zeigte im Tierversuch zentral depressive Wirkungen und Muskelrelaxation. Eine japanische
Arbeitsgruppe konnte zeigen, daß diese Effekte durch andere Prinzipien als die bisher aus dieser Droge isolierten Alkaloide, wie z. B. Magnocurarin, hervorgerufen werden müssen. Als solche wurden aus der alkalilöslichen Fraktion zwei isomere Dihydroxydiallyldiphenyle, Magnolol (XCI) und Honokiol (XCII),

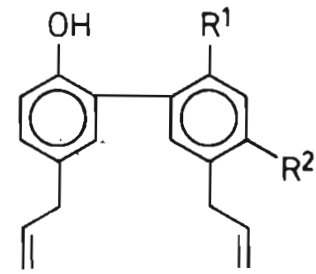

$X C I$ Magnolol $\left(R^{1}=O H, R^{2}=H\right)$ $X$ CII Honokiol $\left(R^{1}=H_{1} R^{2}=O H\right)$

isoliert [172]. Während MagnotQl schon recht lange bekannt war, wurde Honokiol 1972 erstmals in Magnolia officinalis und $M$. obovata gefunden [173]; beide Substanzen wurden unlängst auch in $\mathrm{M}$. grandiflora nachgewiesen [174]. Der Index für die muskelrelaxierende Wirkung der beiden Biphenyle wurde durch Hängedauer von Mäusen an einem Netzwerk untersucht und mit Referenz-Substanzen verglichen: Die $E D_{50}$-Werte (i.p.) betrugen in $\mathrm{mg} / \mathrm{kg}$ : Chlorpromazin 9, Mephenesin 106, Magnolol 131, Honokiol 217 und Methocarbamol 236. Mit $75 \mathrm{mg} / \mathrm{kg}$ Magnolol und $150 \mathrm{mg} / \mathrm{kg}$ Honokiol konnte der Spannungsreflex beim Küken inhibiert werden; dieser Effekt ließ sich durch Strychnin antagonisieren.

Aristolochia clematitis (Aristolochiaceae) ist eine der ältesten Heilpflanzen der Alten Welt. Die in ihrem Rhizom vorkommende Aristolochiasäure (XCV) hat als Phagocytose-Stimulans Eingang in die Therapie gefunden. Diese Nitrophenanthrencarbonsäure kommt zusam- 
men mit ihrem Nitro-freien Analogon, Aristolinsäure (XCIII), und deren Methylester (XCIV) in Aristolochia indica vor, deren Wurzeln in der Volksmedizin Indiens als Emmenagogum und Abortivum Verwendung finden [175]. Nach-<smiles></smiles>

XCIII Aristolinsäure $\left(R^{\prime}=R^{2}=H\right)$

$X C I V$ Aristolinsäuremethylester $\left(\mathrm{R}^{1}=\mathrm{CH}_{3}, \mathrm{R}^{2}=\mathrm{H}\right)$

$\mathrm{XCV}$ Aristolochiasäure $\left(R^{1}=\mathrm{H}, \mathrm{R}^{2}=\mathrm{NO}_{2}\right)$

dem die starke abortive Wirkung verschiedener Extrakte aufgefallen war [176], wurden Aristolinsäure und ihr Methylester in dieser Richtung am Indian Institute of Experimental Medicine in Calcutta eingehend untersucht [177]. An Mäusen wirkte der Ester $100 \%$ ig abortiv, wenn er am 6 . oder 7. Schwangerschaftstag mit $60 \mathrm{mg} / \mathrm{kg}$ oral gegeben wurde, dagegen nur zu 25 bzw. $20 \%$ bei gleicher Dosis am 10. oder 12. Tag [178]. Die freie Säure wurde am Kaninchen untersucht: Orale Gabe von $60 \mathrm{mg} / \mathrm{kg}$ am 9.- Schwangerschaftstag bewirkte bei $65 \%$, mit $90 \mathrm{mg} / \mathrm{kg}$ bei $80 \%$ der Tiere Verlust der Foeten. Signifikante Veränderungen des Blutbildes oder sonstige toxische Effekte wurden nicht beobachtet [179].

Schizandra chinensis ist eine Magnoliacee, deren Früchte in der chinesischen Medizin unter dem Namen "Wu-wei-zi“ bei Dysenterie und Erkältungen Anwendung finden. Ein alkoholischer Extrakt der Fruchtkerne erniedrigt die SerumGlutamin-Pyruvat-Transaminase (SGPT) bei Versuchstieren und Patienten mit Hepatitis. Aus diesem Extrakt wurde Schizandrin $B(\mathrm{XCVI})$ isoliert, ein neuer Vertreter der Bisbenzocyclooctadiene, einer Untergruppe der Lignane, zu der die Schizandrine, Gomisine, Stegane und

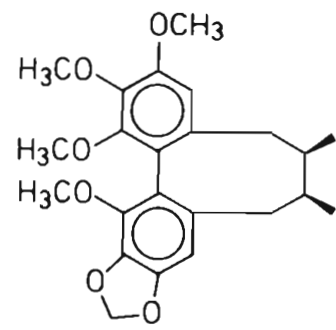

XCVI Schizandrin B

Kadsurine gehören. Chinesische Autoren berichteten über eine Leber-protektive Wirkung der Substanz [180]. Durch $\mathrm{CCl}_{4}$-Intoxikation erhöhte SGPT-Werte wurden bei Mäusen durch orale Schizandrin-B-Gaben vor oder nach der Intoxikation erniedrigt. Die Substanz bewirkt eine Lebervergrößerung sowohl bei $\mathrm{CCl}_{4}$-behandelten Mäusen als auch bei Kontrolltieren. In partiell hepatektomierten Mäusen wird das restliche Lebergewebe nach oraler Schizandrin BGabe schneller regeneriert. Der Effekt von Schizandrin B auf die PentobarbitalSchlafzeit ist biphasisch, je nach Länge des Intervalls zwischen Verabreichung der Droge und Pentobarbital, und wird auf eine Inhibierung oder Induktion mikrosomaler Leber-Enzyme zurückgeführt. Schizandrin B erhöht deutlich die 
Resistenz von Mäusen gegen toxische Effekte des Digoxins und Indomethacins. Die Toxizität der Substanz ist gering; Lebervergrößerungen gehen wenige Tage nach Absetzen auf die ursprüngliche Größe zurück. In zwei weiteren chinesischen Arbeiten wird über die Senkung erhöhter SGPT-Werte durch ähnlich strukturierte Schizandrine (Wuweizisu A-C und Wuweizichun A, B aus Schizandra chinensis [181], Schisantherine A-D aus S. sphenanthera [182]) berichtet.

\section{Rückblick}

In diesem Uberblick wurden 51 pharmakologisch interessante Naturstoffe bzw. Naturstoffgruppen vorgestellt. Davon stammen 43 aus höheren Pflanzen (Samenpflanzen), 5 aus dem übrigen Pflanzenreich und 3 aus tierischen Organismen. Die chemische Verteilung gibt folgendes Bild: 15 sind Alkaloide und andere $\mathrm{N}$-haltige Verbindungen, 10 Terpenoide, 4 Sauerstoff-Heterocyclen, 6 Glykoside und 6 besitzen andere Strukturen. Was die Wirkungsrichtung anbelangt, haben 9 Verbindungen eine Herz-Kreislauf-Wirkung, 7 wirken auf das ZNS, 6 sind antiinflammatorisch aktiv, 5 kontrazeptiv oder anderweitig gynäkologisch, je 4 zeigen Spasmolyse, Muskelrelaxation, Antiulcer-Wirkung, antiatherosklerotische Eigenschaften (hypocholesterinämisch, hypolipämisch, fibrinolytisch, Thromobozytenaggregationshemmend) oder haben Einfluß auf den körpereigenen oder Arzneimittel-Stoffwechsel. 20 Verbindungen stammen aus Pflanzen, die volksmedizinische Verwendung fanden.

\section{Literatur}

1. Baumgarth, M.: Chemiker-Ztg. 97, 217 (1973).

2. Tamm, C.: in [16], 32.

3. Cordell, G. A.: in [16], 54 .

4. Cordell, G. A., N. R. Farnsworth: J. Natur. Prod. 40, 1 (1977).

5. Wall, M. E., M. C. Wani; Ann. Rev. Pharmacol. Toxicol. 17, 117 (1977).

6. Farnsworth, N. R., A. S. Bingel: in [16], 1.

7. Reis-Arndt, E.: Pharmazeut. Industrie 37, 223 (1975).

8. Reis-Arndt, E.: Pharma-Dialog Nr. 57 (1978).

9. Fitoterapia 99 (1979).

10. Thies, W. P.: in [16], 266

11. Pars, H. G., R. K. Razdan, J. F. Howes: in Simmons, A. B. (Ed.), Advances in Drug Research, Vol. 11, 97, New York, 1977, Academic Press.

12. Mechoulam, R., E.A. Carlini: Naturw. 65. 174 (1978).

13. Bernardi, L.: Arzneimittel-Forsch. 29, 1204 (1979)

14. Johne, S.: Pharmazic 34, 279 (1979).

15. Farnsworth, N. R., G. A. Cordell: Lloydia 39,420 (1976).

16. Wagner, H., P. Wolff (Eds.): New Natural Products and Plant Drugs with Pharmacological, Biological or Therapeutical Activity, Berlin, Heidelberg, New York 1977, Springer-Verlag.

17. Achenbach, $\mathrm{H}$.: in [16], 108.

18. Sticher, O.: in [16], 137.

19. Shibata, S.: in [16], 177.

20. Govindachari, T. R.: in [16], 212.

21. Takemoto, T., N. Takagi, T. Nakajima, K. Koike: Yakugaku Zasshi 95, 176 (1975) [C.A. 82, 152211 (1975)].

22. Takemoto, T., T. Nakajima, S. Arihara, K. Koike: Yakugaku Zasshi 95, 326 (1975) [C.A. 83, 43706 (1975)].

23. Takemoto, T., K. Koike, T. Nakajima, S. Arihara: Yakugaku Zasshi 95, 448 (1975) [C.A. 83, 97875 (1975)].

24. Shinozaki, H., I. Shibuya: J. Neuropharmacology 13, 665 (1974).

25. Biscoe, T. J., R. H. Evans, P. M. Headley, M. Martin, J. C. Watkins: Nature 255, 166 (1975). 
26. Menon, I. S.: Brit. Med. J. I, 845 (1969 u. 2, 421 (1970).

27. Augusti, K. T., M. E. Benaim, H. A. Dewar, R. Virden: Atherosclerosis 2/, 409 (1975).

28. Virtanen, A. I., E. J. Matikkala: Acta Chem. Scand. 13, 623 (1959).

29. Agarwal, R. K., H. A. Dewar, D. J. Newell, B. Das: Atherosclerosis 27, 347 (1977).

30. Genest, K., B. B. Coldwell, D. W. Hughes: J. Pharm. Pharmac. 20, 102 (1968) u. 21, 176 (1969).

31. Lindberg, P., R. Bergman, B. Wickberg: J. Chem. Soc. Perkin I 684 (1977).

32. Hatfield, G. M., J. P. Schaumberg: Lloydia 38,489 (1975).

33. Carlsson, P. A. E., P. L. Lindberg, B. V. Wickberg (Astra): DOS 2625110 [C.A. 87, 134025 (1977)].

34. Wickberg, B. V.: Acta Pharm. Suecica 14 (Suppl.) 30 (1977).

35. Tottmar, O., P. Lindberg: Acta Pharmacol. Toxicol. 40, 476 (1977) [C.A. 87, 63457 (1977)].

36. Dhar, K. L., C. K. Atal: Indian J. Chem. S, 588 (1967).

37. Chandhoke, N., S. Gupta, S. Dhar: Indian J. Pharm. Sci. 40, 113 (1978).

38. Konno, C., T. Taguchi, M. Tamata, H. Hikino: Phytochemistry 18, 697 (1979).

39. Tamada, M., K. Endo, H. Hikino: Planta medica 34, 291 (1978).

40. Tamada, M., K. Endo, H. Hikino, C. Kabuto: Tetrahedron Lett. 873 (1979).

41. Tamada, M., K. Endo, H. Hikino: Heterocycles /2, 783 (1979).

42. Gilbert, B. et al.: J. Amer. Chem. Soc. 86, 694 (1964).

43. Ferrari, G., C. Casagrande: Il Farmaco, Ed. Sci. 25, 449 (1970).

44. Casagrande, C.: Abstr. Papers, Amer. Chem. Soc. 167 Meet. MEDI 31 (1974).

45. Casagrande, C., L. Canonica: J. Chem. Soc. Perkin I 1647 u. 1652 (1975).

46. Kametani, T., K. Takahashi, K. Ogasawara, C. van Loc, K. Fukumoto: Coll. Czech. Chem. Commun. 40, 712 (1975).

47. Ferrini, R., G. Miragoli: Boll. Soc. It. Biol. Sper. SO, 1629 u. 1635 (1974).

48. Buffa, B., G. Costa, P. Ghirardi: Curr. Ther. Res. 16, 621 (1974).
49. Chaumontet, M., M. Capt, P. Gold-Aubert: Arzneim.-Forsch. 28 (II), 2119 (1978).

50. Yusupov, M. K., A. S. Sadykov: Uzb. Khim. Zh. S, 49 (1961) [C.A. 56, 8839 (1962)].

51. Yusupov, M. K., A. S. Sadykov: Zh. Obshch. Khim. 34, 1672 (1964) [C.A. 61, 5962 (1964)].

52. Yusupov, M. K., A. S. Sadykov: Khim. Prir. Soedin 350 (1976) [C.A. 86, 43862 (1977)].

53. Yusupov, M. K. et al.: Coll. Czech. Chem. Commun. 42, 1581 (1977).

54. Emel'yanov, L. S., S. K. Nasirov: Farmakol. Toksikol. (Moskau) 37, 664 (1974) [C.A. 82, 118903 (1975)].

55. Persinos Perdue, G., R. N. Blomster, D. A. Blake, N. R. Farnsworth: J. Pharm. Sci. 68 , 124 (1979).

56. Platonova, T. F., A. D. Kuzovkov, P. S. Massagetov: Zhur. Obshchei Khim. 23, 880 (1953) [C.A. 48, 3987 (1954)].

57. Kamalitdinov, D., S. Ikandarov, S. Y. Yunusov: Khim. Prir. Soedin 5, 409 (1969) [C. A. 72, 75653 (1970)].

58. Platonova, T. F., A. D. Kuzovkov, N. Sheinker: Zhur. Obshchei Khim. 26, 2651 (1956) [C.A. 51, 5102 (1957)].

59. Sheinker, Y. N.: C.A. 53, 22734 (1959).

60. Shamma, M., J. L. Moniot: Chem. Commun. 1065 (1971).

61. Tomita, M., Y. Sasaki: Chem. Pharm. Bull. 1, 105 (1953); 2, 89 (1954), 3, 178, 250 (1955).

62. Drugs of the Future 4, 481 (1979).

63. Moza, B. K.: Indian J. Chem. 5, 281 (1967).

64. Ray, A. B., S. Chattopadhyay, R. M. Tripathi, S. S. Gambhir, P. K. Das: Planta medica 35, 167 (1979).

65. Tomita, M., E. Fumita: Pharm. Bull (Japan) 2, 372 (1954) [C.A. 50, 12085 (1956)].

66. Zarembo, J. E., B. Douglas, J. Valenta, J. A. Weisbach: J. Pharm. Sci. 63, 1407 (1974).

67. Meyer, W. E., J. A. Coppola, L. Goldman: J. Pharm. Sci. 62, 1199 (1973).

68. Kupchan, S. M., A. Bright, E. Macko: J. Pharm. Sci. 52, 598 (1963).

69. Gorman, M., N. Neuss, N. J. Cone, J. A. Deyrup: J. Amer. Chem. Soc. 82, 1142 (1960).

70. Mehrotra, P. K., V. P. Kamboj: Planta medica 33,345 (1978).

71. Das, B., K. Biemann: Tetrahedron Lett. 2483 (1966). 
72. Bhattacharya, S. K., A. B. Ray, S. R. Guha: Pharmacol. Res. Commun. 8, 159 (1976).

73. Sakai, S.: Heterocycles 4, 131 (1976).

74. Aimi, N., K. Yamaguchi, S. Sakai, J. Haginiwa, A. Kubo: Chem. Pharm. Bull. 26, 3444 (1978).

75. Murayama, S., M. Harada, Y. Ozaki, T. Suzuki: Japan J. Pharmacol. Suppl. 23, 21 (1973).

76. Harada, M., Y. Ozaki, M. Sato: Chem. Pharm. Bull. 22, 1372 (1974).

77. Harada, M., Y. Ozaki: Chem. Pham. Bull. 24, 211 (1976).

78. Harada, M., Y. Ozaki: Chem. Pharm. Bull. 26,48 (1978).

79. Culvenor, C. C. J., J. A. Edgar, M. V. Jago, A. Outteridge, J. E. Peterson, L. W. Smith: Chem.-Biol. Interactions 12, 299 (1976).

80. Pomeroy, A. R., C. Raper: Arch. int. Pharmacodyn. 199, 5 (1972).

81. Atal, C. K.: J. Natur. Prod. 4l, 313 (1978).

82. Gupta, O. P., M. M. Ali, B. J. R. Ghatak, C. K. Atal: Indian J. Exp. Biol. 15, 220 (1977) [C. A. 87, 15875 (1977)].

83. Gupta, O. P., G. B. Singh, B. J. R. Ghatak, C. K. Atal: Indian J. Exp. Biol. 14, 282 (1976).

84. Gupta, O. P., M. M. Ali, B. J. R. Ghatak, C. K. Atal: Indian ]. Exp. Biol. 14, 34 (1976) [C. A. 84, 115633 (1976)].

85. Gupta, O. P., M. M. Ali, B. J. R. Ghatak, C. K. Atal: Indian J. Exp. Biol. 15, 1001 (1977) [C. A. 88,182785 (1978)].

86. Gupta, O. P., G. B. Singh, C. K. Atal: Arzneim.-Forsch. 29, 1715 (1979).

87. Goosen, A.: J. Chem. Soc. 3067 (1963).

88. Van Eijk, J. I.., M. H. Radema: Pharm. Weekbl. 111, 1285 (1976).

89. Gerrans, G. C., J. Harley-Mason: J. Chem. Soc. 2202 (1964).

90. Lindner, E., J. Kaiser, L. Schacht: Arzneim.Forsch. 26, 1651 (1976).

91. Drugs of the Future 2, 365 (1977).

92. Komoda, Y., S. Kaneko, M. Yamamoto, M. Ishikawa, A. Itai, Y. Iitaka: Chem Pharm. Bull. 23, 2464 (1975).

93. Cariello, L., S. Crescenzi, G. Prota, L. Zanetti: Tetrahedron 30, 4191 (1974).

94. Adams, J. H., M. R. B. Blair Jr., B. H. Takman: Arch. Int. Pharmacodyn. 224, 275 (1976).
95. Drugs of the Future 3, 216 (1978).

96. Yeung, H. W., Y. C. Kong, W. P. Lay, K. F. Cheng: Planta medica 31, 51 (1977).

97. Cheng, K. F., C. S. Yip, H. W. Yeung, Y. C. Kong: Experientia 35, 571 (1979).

98. Drugs of the Future 2, 597 (1977).

99. Del Castillo, J., M. Anderson, G. M. Rubot tom: Nature 253, 365 (1975).

100. Rice, K. C., R. S. Wilson: J. Med. Chem. 19, 1054 (1976).

101. Fllestad, G. A.: J. Org. Chem. 27, 1855 (1962).

102. Rybalko, K. S., L. Dolejs: Coll. Czech. Chem. Commun. 26, 2909 (1961).

103. Rodriguez de Vera, C., S. Pcran Mesa, A. M. De Diego, A. Villar del Fresno: Boll. Chim. Farm. 1/5, 445 (1976).

104. Romo de Vivar, A., C. Guerrero, F. Diaz, A. Ortega: Tetrahedron 26, 1657 (1970).

105. Valdés, R., F. Córdoba: Agents and Actions, 5, 64 (1975).

106. Bisarya, S. C., S. Dev: Tetrahedron 24, 3861 u. 3869 (1968).

107. Kulshreshtha, D. K., R. P. Rastogi: Phytochem. 14, 2237 (1975).

108. Kar, K., V. N. Puri, G. K. Patnaik, R. N. Sur, B. N. Dhawan, D. K. Kulshreshtha, R. P. Rastogi: J. Pharm. Sci. 64, 258 (1975).

109. Puri, V. N., K. Kar, G. K. Patnaik, B. N. Dhawan, D. K. Kulshreshtha, R. P. Rastogi: Indian J. Exp. Biol. 13, 369 (1975)

110. Tandon, J. S., M. M. Dhar, S. Ramakumar, K. Venkatesan: Indian J. Chem. I5B, 880 (1977).

111. Bhat, S. V., B. Bajwa, H. Dornauer, N. J. de Souza, H. W. Fehlhaber: Tetrahedron Lett. 1969 (1977).

112. Lindner, E., A. N. Dhadwalla, B. K. Bhattacharya: Arzneim.-Forsch. 28, 284 (1978).

113. Ogiso, A. et al.: Chem. Pharm. Bull. 26, 3117 (1978).

114. Kitazawa, E. et al,: Tetrahedron Lett. 1117 (1979).

115. Haefliger, W., D. Hauser: Helv. Chim. Acta S6, 2901 (1973).

116. Wiesinger, D., H. U. Gubler, W. Haefliger, D. Hauser: Experientia 30, 135 (1974).

117. Haefliger, W., D. Hauser: Helv. Chim. Acta 58,1629 (1975).

118. Haefliger, W., Z. Kis, D. Hauser: Helv. Chim. Acta 58, 1620 (1975). 
119. Satyavati, G. V., M. K. Raina, M. Sharma, (Eds.): Medicinal Plants of India, Vol. I, p 269, New Delhi 1976, Indian Council of Medical Research.

120. Patil, V. D., U. R. Nayak, S. Dev: Tetrahe dron 28, 2341 (1972).

121. Kapoor, N. K., S. Nityanand: Indian J. Biochem. Biophys. 15, 77 (1978).

122. Mester, L., M. Mester, S. Nityanand: Planta medica 37, 367 (1979).

123. Adams, R., T. A. Geissman, J. D. Edwards: Chem. Rev. 60, 555 (1960).

124. Berardi, L. C., L. A. Goldblatt: Toxic Constituents of Plant Foodstuffs, New York 1969, Academic Press.

125. Datta, S. C., V. V. Murti, T. R. Seshadri: Current Sci. 41, 545 (1972).

126. Drugs of the Future 4, 338 (1979).

127. Méd. et Hyg. 37, 831 (1979) [Selecta 2517 (1979)].

128. Drugs of the Future 3, 550 (1978).

129. Singh, G., G. V. Nair, K. P. Aggarval: Chem. and Ind. 1294 (1954).

130. King, F. E., J. R. Hously, T. J. King: J. Chem. Soc. 1392 (1954).

131. Jamwal, K. S., M. L. Sharma, N. Chandhoke, B. J. R. Ghatak: Indian J. Med. Res. 60, 763 (1972) [C. A. 77, 160212 (1972)].

132. Thakur, R. S., S. C. Bagadia, M. L. Sharma: Experientia 34, 158 (1978).

133. Chem. Ind. 29, 555 (1977).

134. Shoeb, A., M. D. Manandhar, R. S. Kapil, S. P. Popli: Chem. Commun. 281 (1978).

135. Stout, G. H., M. M. Krahn, P. Yates, H. B. Bhàt: Chem. Commun. 211 (1968).

136. Hostettmann, K., H. Wagner: Phytochemistry 16, 821 (1977).

137. Shankaranarayan, D., C. Gopalakrishnan, L. Kameswaran: Arch. int. Pharmacodyn. 239, 257 (1979).

138. Endo, A., M. Kuroda, Y. Tsujita: J. Antibiot. (Tokyo) 29, 1346 (1976).

139. Brown, A. G., T. C. Smale, T. J. King, R. Hasenkamp, R. H. Thompson: J. Chem. Soc. Perkin I, 1165 (1976).

140. Drugs of the Future 3, 662 (1978).

141. Betteridge, D. J., J. P. D. Reckless, W. Krone, D. J. Galton: Lancet 1342 (1978, II).

142. Brown, M. S., J. R. Faust, J. I.. Goldstein, I. Kaneko, A. Endo: J. Biol. Chem. 253, 1121 (1978).
143. Flanders, L. E., P. Palicharla, N. Nicholson: Fed. Proc. 33, Abstr. 2803 (1. März 1979).

144. Gonzáles, A. G., C. G. Fráincisco, R. Freire, R. Hernández, J. E. Salazar, E. Suárez: Phytochem. Is, 344 (1976).

145. Darias, V., A. G. Gonzáles, J. N. Boada, M. Feria, F. Martorell: Il Farmaco (Ed. Sci.) 33, 460 (1978).

146. Hirata, T., T. Suga: Z. Naturforsch. C 32, 731 (1977).

147. Suga, T., T. Hirata, M. Odan: Chem. Lett. 547 (1972).

148. Hirata, T., T. Suga: Bul]. Chem. Soc. Japan 51,842 (1978).

149. Suga, T., T. Hirata: Bull. Chem. Soc. Japan 51,872 (1978).

150. Sih, C. J., P. R. Ravikumar, F.-C. Huang, C. Buckner, H. Whitlock jr.: J. Am. Chem. Soc. 98, 5412 (1976).

151. Koshiyama, H., M. Hatori, H. Ohkuma, F. Sakai, H. Imanishi, M. Ohbayashi, H. Kawaguchi: Chem. Pharm. Bull. 24, 169 (1976).

152. Sakai, F., H. Ohkuma, H. Koshiyama, T. Naito, H. Kawaguchi: Chem. Pharm. Bull. 24, 114 (1976).

153. Drugs of the Future 3, 567 (1978).

154. Mizuno, K., M. Tsujino, M. Takada, M. Hayashi, K. Atsumi, K. Asano, T. Matsuda: J. Antibiotics 27, 775 (1974).

155. Yoshioka, H., K. Nakatsu, M. Hayashi, K. Mizuno: Tetrahedron Lett. 4031 (1975).

156. Iwata, H., H. Iwaki, T. Masukawa, S. Kasamutso, H. Okamoto: Experientia 33, 502 (1977).

157. Gregson, R. P., R. J. Quinn, A. F. Cook (Hoffmann-La Roche AG): DOS $2833887=$ Eur. Pat. 770 [C. A. 91, 39792 (1979)].

158. Tanaka, S., Y. Ikeshiro, M. Tabata, M. Konishima: Arzneim.-Forsch./Drug. Res. 27 (II), 2039 (1977)

159. Bentley, R. K., D. Bhattacharjee, E. R. H. Jones, V. Thaller: J. Chem. Soc. (C) 685 (1969).

160. Bohlmann, F., C. Arndt, H. Bornowski, K.M. Kleine: Chem. Ber. 94, 958 (1961).

161. Bohlmann, F., U. Niedballa, K.-M. Rode: Chem. Ber. 99, 3552 (1966).

162. Fukuzawa, A., E. Kurosawa, T. Irie: Tetrahedron Lett. 3 (1972).

163. McDonald, F. J., D. C. Campbell, D. J. Vanderah, F. J. Schmitz, D. M. Washecheck, J. 
E. Burks, D. van der Helm: J. Org. Chem. 40, 665 (1975).

164. Irie, T., M. Izawa, E. Kurosawa: Tetrahedron 26, 851 (1970).

165. Irie, T., M. Suzuki, T. Masamune: Tetrahedron 24, 4193 (1968).

166. Cameron, A. F., K. K. Cheung, G. Ferguson, J. M. Robertson: Chem. Commun. 683 (1965).

167. Masamune, T., H. Murare, H. Matsue, A. Murai: Bull. Chem. Soc. Jap. 52, 135 (1979).

168. Kaul, P. N., S. K. Kulkarni, E. Kurosawa: ]. Pharm. Pharmacol. 30, 589 (1978).

169. Kaul, P. N., S. K. Kulkarni, L. R. Whitfield: Pharmacologist 20, 267 (1978).

170. Kaul, P. N., S. K. Kulkarni: J. Pharm. Sci. 67, 1293 (1978).

171. Mitsui, S., S. Kobayashi, H. Nagahori, A. Ogiso: Chem. Pharm. Bull. 24, 2377 (1976).

172. Watanabe, K., H. Y. Watanabe, Y. Goto, N. Yamomoto, M. Yoshizaki: Jap. J. Pharmacol. 25, 605 (1975).

173. Fujita, M., H. Itokawa, Y. Sashida: Chem. Pharm. Bull. 20, 212 (1972).
174. El Feraly, F. S., W. S. Li: J. Natur. Prod. 41, 442 (1978).

175. Pakrashi, S. C., P. Gosh-Dastidar, S. Basu, B. Achari: Phytochem. 16, 1103 (1977).

176. Pakrashi, A., B. Chakrabarty, A. Dasgupta: Experientia 32, 394 (1976).

177. Drugs of the Future 4, 475 (1979).

178. Pakrashi, A., C. Shaha: Experientia 34, 1192 (1978).

179. Pakrashi, A., B. Chakrabarty: Experientia 34, 1377 (1978).

180. Drugs of the Future 3, 613 (1978).

181. Chen, Y.-Y., Z.-B. Shu, L.-N. Li: Sci. Sin. 19, 276 (1976) [C. A. 86, 29518 (1977)].

182. Sheng, Wu Hua Hsueh Yu Sheng Wu Wu Li Hsueh Pao 8, 333 (1976) [C. A 88, 55007 (1978)].

Anschrift: Dr. M. Baumgarth Pharma-Forschung Naturstoffe E. Merck Postfach 4119 D-6100 Darmstadt 